Proceedings of the Institution of Mechanical Engineers, Part C: Journal of Mechanical Engineering Science

https://journals.sagepub.com/home/pic

Impact factor $=1.386$

(ACCEPTED OCTOBER 15 ${ }^{\text {TH } 2020)}$

\title{
Numerical Investigation of von Karman Swirling Bioconvective Nanofluid Transport from a Rotating Disk in a Porous Medium with Stefan Blowing and Anisotropic Slip Effects
}

\author{
O. Anwar Bég ${ }^{1}$, Muhammad Nomani Kabir ${ }^{2 *}$, Md Jashim Uddin $^{3,4}$, Ahmad Izani Md. Ismail $^{3}$ and Yasser Alginahi ${ }^{5}$ \\ ${ }^{1}$ Professor and Director: Multi-Physical Engineering Sciences Group (MPESG), Aeronautical and Mechanical \\ Engineering, School of Science, Engineering and Environment (SEE), Salford University, Manchester, M54WT, UK \\ Email:O.A.Beg@salford.ac.uk,gortoab@gmail.com \\ ${ }^{2}$ Faculty of Computing, University Malaysia Pahang, 26300 Gambang, Pahang, Malaysia \\ *Corresponding author - email: nomanikabir@ump.edu.my \\ ${ }^{3}$ School of Mathematical Sciences, Universiti Sains Malaysia, 11800, Penang, Malaysia. ahmad_izani@usm.my \\ ${ }^{4}$ American International University-Bangladesh, Banani, Dhaka 1213, Bangladesh \\ Email: jashim_74@yahoo.com \\ ${ }^{5}$ Dept. of Electrical and Computer Engineering, University of Windsor, Windsor, Canada. alginah@uwindsor.ca
}

\begin{abstract}
${ }^{\wedge}$ Now at: Computer Information Systems, Adrian University College, Adrian, Michigan, MI, USA.
ABSTRACT

In recent years, significant progress has been made in modern micro- and nanotechnologies related to applications in micro/nano-electronic devices. These technologies are increasingly utilizing sophisticated fluent media to enhance performance. Among the new trends is the simultaneous adoption of nanofluids and biological micro-organisms. Motivated by bio-nanofluid rotating disk oxygenators in medical engineering, in the current work, a mathematical model is developed for steady convective Von Karman swirling flow from an impermeable power-law radially stretched disk rotating in a Darcy porous medium saturated with nanofluid doped with gyrotactic micro-organisms. Anisotropic slip at the wall and blowing effects due to concentration are incorporated. The nano-bio transport model is formulated using non-linear partial differential equations (NPDEs), which are transformed to a set of similarity ordinary differential equations (SODEs) by appropriate transformations. The transformed boundary value problem is solved by a Chebyshev collocation method. The impact of key parameters on dimensionless velocity components, concentration, temperature and motile microorganism density distributions are computed and visualized graphically. Validation with previous studies is included. It is found that that the effects of suction provide a better enhancement of the heat, mass and microorganisms transfer in comparison to blowing. Moreover, physical quantities decrease with higher slip parameters irrespective of the existence of blowing. Temperature is suppressed with increasing thermal slip whereas nanoparticle concentration is suppressed with increasing wall mass slip. Micro-organism density number increases with the greater microorganism slip. Radial skin friction is boosted with positive values of the power law stretching parameter whereas it is decreased with negative values. The converse response is computed for circumferential skin friction, nanoparticle mass transfer rate and motile micro-organism density number gradient. Results from this study are relevant to novel bioreactors, membrane oxygenators, food processing and bio-chromatography.
\end{abstract}

KEYWORDS: Porous medium, bio-nano-convective slip flow, rotating disk, Stefan blowing, Anisotropic slips, rotating disk membrane oxygenators.

\section{NOMENCLATURE}

$\tilde{b}$

$C$

$C_{w}$

$C_{w}$

$C_{f \bar{r}}$ chemotaxis bioconvection constant $(m)$

nanoparticle volume concentration (-)

nanoparticle volume concentration at the wall (-)

nanoparticle volume concentration at free stream (-)

local skin friction coefficient in $\bar{r}$-direction (- ) 
$C_{f \theta} \quad$ local skin friction coefficient in $\theta$-direction (-)

Da Darcy number (-)

$D_{B} \quad$ Brownian diffusion coefficient $\left(\mathrm{m}^{2} \mathrm{~s}^{-1}\right)$

$D_{n} \quad$ microorganism diffusion coefficient $\left(\mathrm{m}^{2} \mathrm{~s}^{-1}\right)$

$D_{T} \quad$ thermophoretic diffusion coefficient $\left(\mathrm{m}^{2} \mathrm{~s}^{-1}\right)$

$D_{1} \quad$ thermal slip factor $(m)$

$\left(D_{1}\right)_{0} \quad$ constant thermal slip factor $(m)$

$E_{1} \quad$ mass slip factor $(m)$

$\left(E_{1}\right)_{0} \quad$ constant mass slip factor $(m)$

$F_{1} \quad$ microorganism slip factor $(\mathrm{m})$

$\left(F_{1}\right)_{0} \quad$ microorganism slip factor $(\mathrm{m})$

$K \quad$ variable permeability of the porous medium $\left(\mathrm{m}^{2}\right)$

$K_{0} \quad$ constant permeability of the porous medium $\left(\mathrm{m}^{2}\right)$

$f(\eta) \quad$ dimensionless axial stream function $(-)$

$g(\eta) \quad$ dimensionless circumferential stream function $(-)$

Le Lewis number $(-)$

$m \quad$ disk radial power-law stretching exponent $(-)$

$\mathrm{Nb} \quad$ Brownian motion parameter $(-)$

Nt thermophoresis parameter $(-)$

$\mathrm{Nu} u_{\bar{r}} \quad$ local Nusselt number $(-)$

$N n_{\bar{r}} \quad$ local wall motile microorganism number $(-)$

$N_{1} \quad$ variable velocity slip factor for $u$ velocity component, $\left(\mathrm{sm}^{-1}\right)$

$\left(N_{1}\right)_{0} \quad$ constant velocity slip factor for $u$ velocity component, $\left(\mathrm{sm}^{-1}\right)$

$N_{2} \quad$ variable velocity slip factor for $v$ velocity component, $\left(\mathrm{sm}^{-1}\right)$

$\left(N_{2}\right)_{0} \quad$ constant velocity slip factor for $v$ velocity component, $\left(\mathrm{sm}^{-1}\right)$

$n \quad$ density number of motile micro-organisms $(-)$

$n_{w} \quad$ wall motile microorganisms $(-)$

Pe bioconvection Péclet number $(-)$

Pr Prandtl number (-)

$q_{w} \quad$ surface heat flux $\left(\mathrm{Wm}^{-2}\right)$

$\bar{r} \quad$ dimensional radial coordinate along the disk $(m)$

$r \quad$ dimensionless radial coordinate along the disk $(-)$

$R \quad$ reference scale length $(m)$ 


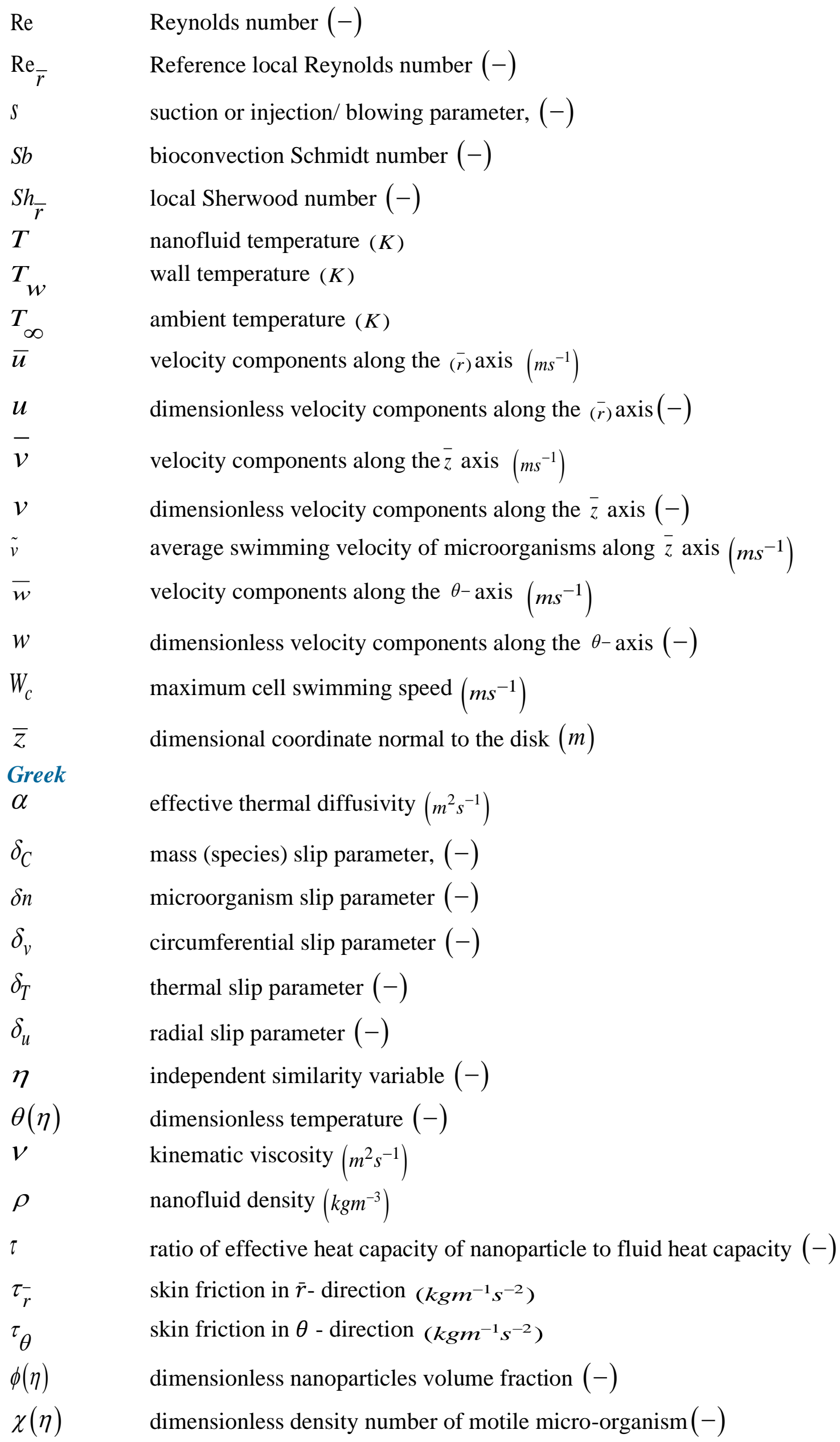


stream function $\left(m^{2} s^{-1}\right)$

angular velocity $\left(m s^{-1}\right)$

\section{INTRODUCTION}

Nanofluids are formed by suspending ultrafine particles (diameter less than $10^{-9} \mathrm{~nm}$ ) in various traditional fluids, namely, water, oil, bioliquids and ethylene glycol etc. Nanoparticles provide a means of improving heat transfer characteristics of the base fluids. Commonly used nanoparticles, such as metal oxide, significantly enhance the thermal properties of the carrier fluids. Nanofluids may be used in hybrid powered machines, transformer oil, motor cooling, solar water heating and nuclear reactor, refrigeration, microscale mixers, coolants and waste heat boiler systems as noted by Minkowycz et al. [1], Zarifi et al. [2], Gravel et al. [3] and Nield and Bejan [4]. The concept of nanofluids was popularized by Choi in 1995 [5]. Two mathematical formulations [6, 7] are widely used for nanofluid transport phenomena. The model proposed by Buongiorno [6] incorporates a nano-particle volume fraction conservation equation in addition to thermophoresis and Brownian motion effects (which are quite similar to the Dufour and Soret terms encountered in thermsolutal convection). On the other hand, the model of Das at el. [7] integrates the fluid properties variation (e.g thermal conductivity, viscosity, electric conductivity) with particle volume fraction, using expressions based on the theory of mixtures. These two popular models are suitable for utilization in nano-bioconvection boundarylayer models. Recent researh works have included a number of analytical and computational techniques for solving various industrial and biological fluid dynamics problems using these models. These studies have considered both external and internal flow for various geometries of relevance to biomedicine. For example, Rahman et al. [8] used numerical methods to compute the flow and heat transfer rates over a sheet considering non-adherence at the boundary. In another article, Acharya et al. [9] analysed the influence of second-order wall slip on nanofluid boundary layer flow under multiple convective boundary conditions with a probabilistic approach. Mahanthesh et al. [10] investigated hydromagnetic non-Newtonian nanofluid dynamics on a convectively heated, radiating and elongating surface with Ohmic and viscous dissipation effects.

More recently, scientists, engineers and applied mathematicians have explored bioconvection flows with emerging applications in bio-inspired technologies. Bioconvection arises owing to the discrepancy in density of the self-propelled micro-organisms (formed by combined swimming of motile microorganisms). Normally, single cell micro-organisms (algae, bacteria etc.) are involved in bioconvective flow, as elaborated by Hwang and Pedley [11]. Gyrotactic micro-organisms (e.g. Chlamydomonas monas and Peridinium gatunense) are able to swim in water against gravity. After developing bioconvection, their swimming direction is controlled by torques. Whereas motile microorganisms are self-propelled, nanoparticles are not. Brownian motion and thermophoresis exert specific forces to cause movement of nanoparticles whereas different stimuli such as chemical attraction, light, magnetic field or gravity are responsible for the movement of micro-organisms. The motile micro-organisms are added to increase mass transfer, micro-scale mixing for improving the stability of the nanofluid and preventing nanoparticle agglomeration. Bioconvection inside nanofluids is a promising research area due its wide range of applications in various fields. Examples include biomicro-systems as discussed by Tsai et al. [12], enzymatic bio-sensors as considered by Li et al. [13], and magnetic bioseparation microscale devices as described by Munir et al. [14]. Bioconvection patterns are generated by upward swimming microorganisms which possess a higher density than water. The mechanism of bioconvection can be exploited in producing ecological fuels, bio-coatings and designing fuel systems. Relevant studies include Chisti [15] on bioethanol, Satyanarayana et al. [16] on PEM fuel cells [16], Aneja et al. [17] on magnetic nano-bio solar collector coatings and Roncallo et al. [18] on photo-bioreactors. Many numerical studies of combined nanofluid and bioconvection transport have been reported recently. $\mathrm{Xu}$ et al. [19] explored nanofluid flow from an extending surface containing gyrotactic microorganism. Raees et al. [20] analysed bioconvective flow between two parallel plates where one is moving and the other remains static, deploying a homotopy analysis method and the Mathematica package. Siddiqa et al. [21] conducted a study on nanofluid bioconvective flow from an upright cone with undulating surface, noting that both amplitude of the 
wavy surface of the cone and cone semi-vertex angle significantly modify heat and nano-particle mass transfer coefficients and the motile microorganism density coefficient.

The bioconvection mechanism can also be used to enhance species diffusion processes which are critical in membrane oxygen bioreactors among other biomedical devices which are reviewed in Taskin et al. [22]. Bioreactor flows require careful computation of characteristics of heat, mass and micro-organism transfer. In rotating disk bioreactors and oxygenators, a key flow regime is viscous transport from a permeable/impermeable rotating disk. This application has been lucidly reviewed by Germbode et al. [23]. This flow regime also features in chromatography, spin coating systems, pharmaceutical manufacturing processes etc. Other applications including thermal therapies for cancer have been reviewed in Gaber and Mohamed [24]. Von Kármán [25] initiated theoretical studies of viscous incompressible flow from a rotating disk. Shevchuk [26] reviewed many mathematical studies of flow, heat and mass transfer problems associated with rotating disk configurations. Von Kármán's model has been extended to consider many more complex flows. Mishra et al. [27] extended the single disk flow problem to a thermo-fluid dual disk scenario with a Catteano-Christov heat flux model for thermal relaxation effects. Bég et al. [28] used Adomian computation to investigate the asymmetric micropolar flow in a bionic dual disk system with transpiration effects at the disk surfaces. Several authors have also examined nanofluid boundary layer flows from rotating disks and other uniform geometries e.g. spheres, ellipses, cones etc. Yin et al. [29] studied heat transfer in nanofluid flow from a rotating disk considering a uniform extending rate along radial axis. Bég et al. [30] analysed the unsteady nanofluid at the stagnation point on a rotating sphere using homotopy methods. Kadir et al. [31] employed Adomian decomposition to simulate the nonlinear gyrotactic bioconvection nanofluid flow from a spinning stretchable disk as a model of a rotating disk bioreactor. All these studies confirmed the significant modification in radial, tangential and axial flow fields and heat and mass diffusion with rotational effects.

Porous media also find many applications in modern biomedical and manufacturing technologies. These include low-density lipoprotein transport across arterial tissues, drug delivery and biomass transport in tissue regeneration, insulation mechanisms for hyperthermia etc. Excellent reviews of the applications related to various organs and cells have been given by Vafai [32] and Khaled and Vafai [33]. Porous media may also feature in rotating disk oxygenators and bioreactors as they provide an efficient filtration mechanism and also can be used for culturing micro-organisms [34]. Thermo-solutal transport in saturated porous media has stimulated considerable attention in engineering analysis. Interesting studies (which usually feature the Darcy viscous-dominated drag force model or the nonDarcy formulation for inertial effects), in this regard, include coupled heat and mass transfer from vertical surfaces in permeable media [35, 36], micropolar plume dynamics in geological porous media [37], geothermal reactive duct flows in non-Darcy media with mixed boundary conditions [38] and biomagnetic oscillatory (pulsatile) hemodynamics in diseased arteries [38]. Several investigators have also addressed the dynamics of Newtonian and non-Newtonian fluids from a rotating disk to a porous medium substrate. Attia [40] studied the steady viscous Newtonian flow and thermal convection from a rotating disk to a high permeability porous medium. Bég et al. [41] used numerical quadrature to compute the pseudoplastic and dilatant fluid flow from a rotating disk to a Darcy-Forchheimer porous material, with applications in biopolymer spin coating. Hayat et al. [42] employed an optimal homotopy analysis method to simulate the carbon-based nanofluid flow from a rotating disk in a nonDarcy medium. They considered both single- and multi-wall carbon nanotubes.

In certain medical device flows, the fluid can slip at the surface of the boundary. In these situations, the classical "no slip" boundary conditions become unrealistic and it is necessary to incorporate wall slip conditions. When saturated porous media are present, fewer molecules will exchange momentum with the fiber, and the hydrodynamic drag on the fiber will be reduced due to momentum slip. However, slip-flow can be advantageous also since it enhances the single fiber capture efficiency of nanoparticles on the fibers and this may be exploited in novel rotating disk bioreactor designs [43, 44]. Experimental as well as theoretical investigations conducted show that slip phenomena arise in 
many other applications also which include biological pumping [45] and rotating microchannels [46]. Slip also arises on hydrophobic and superhydrophobic walls in fuel cells.

Wall mass flux also arises in many industrial and medical applications. Species transfer through a porous wall arises in for example evaporation or condensation during paper drying, as elaborated in Lienhard and Lienhard [47]. Depending on the water content and temperature of wet papers, mass transfer can produce a blowing effect, which is caused by diffusion of species. The diffusion creates a bulk motion of the fluid, introducing an additional motion that is termed as Stefan blowing. This blowing effect is not the same as the mass injection resulting from transpiration for permable surfaces. It has also been utilized in rotating biofilm reactors [48]. The blowing effects for various flow configurations has been investigated by many researchers. Hajmohammadi et al. [49] studied Stefan blowing effects on copper/silver -water nanofluid flow from a stretching sheet. Lattif et al. [50] investigated Stefan blowing effects on a nanofluid doped with microorganism in swirling flow from a solid rotating stretchable disk. Uddin et al. [51] conducted an investigation on the multiple-slip and blowing effects on natural convective flow of bio-nanofluid. Zohra et al. [52] investigated anisotropic slip- and blowing properties of MHD bio-nanofluid flow over a rotating cone and Ragui et al. [53] examined convective phenomena due to double-diffusion in a porous annulus.

In general, nano-bio-convective phenomena are governed by a system of non-linear partial differential equations associated with a variety of physical boundary conditions (slip, convective, chemical reactions, radiative-convective etc). Due to the strong nonlinearity of problems in viscous fluid dynamics, analytical solutions are generally not possible. Hence, recourse must be made to semianalytical/numerical or fully computational methods. Some popular numerical methods are finite difference (FDM), finite volume (FVM), finite element (FEM), boundary characteristics orthogonal polynomials (BCOPs), boundary element method (BEM) etc. Some of the widely used semi-analytical methods which comprise power series expansions includes homotopy perturbation method (HPM), Adomian decomposition method (ADM), homotopy analysis (HAM), variational iteration Method (VIM), differential transform method (DTM) etc. For details of these (and other) methods readers are referred to Bég et al. [54]. The Chebyshev spectral collocation method (CSCM) has a superior convergence rate, requires less grid points to obtain correct results compared to other commonly used methods, namely RK45, FDM, FEM and FVM. CSCM provides exponential convergence rates whilst FDM, FEM and FVM provide linear convergence, as noted by Canuto [55]. The foremost advantage of spectral methods lies in their correctness for a given number of unknowns. CSCM has been widely applied in many scientific areas. Examples include computational fluid dynamics (Kaladhar et al. [56], electrodynamics (Belgacem and Grundmann [57]), free vibration analysis of rotating and nonrotating Timoshenko beams (Ma'en and Butcher [58], magnetohydrodynamics (Aly and Sayed [59]), fractional diffusion equations (Agarwal and El-Sayed [60]), magneto-bioconvection wedge flow (Uddin et al. [61]), non-Newtonian flow (Tian et al. [62]), 3-D transient radiative-conductive heat transfer (Sun et al. [63]), thermally stratified fluid in porous medium (Vasu et al. [64]), 3-D Helmholtz-type equations (Bai et al. [65]), electrohydrodynamic ion drag pump flows (Bég et al. [66]) and ferromagnetic nano-coating dynamics (Shamshuddin et al. [67]). In spite of the high precision and efficacy of the method, it has not been used extensively in nonlinear bioconvection nanofluid dynamics. In this study, the Chebyshev spectral collocation method is employed in a MATLAB symbolic environment.

Inspection of the scientific literature has revealed that in simulations of bioconvection nanofluid flow from a rotating disk with power-law radial stretching immersed in a Darcy porous medium, researchers have not taken into account the normal interfacial velocity associated with mass diffusion and multiple slips effects. This is addressed for the first time in the current study. Extensive details of the mathematical model are presented. Visualization of computations is included for the influence of a number of parameters including Darcy number, microorganism slip, mass (nanoparticle) slip, circumferential momentum slip, radial momentum thermal slip and Stefan blowing/suction. The inclusion of multiple anisotropic slip effects also constitutes a novelyu of the present work. Validation 
with special cases from the literature is also conducted. The computations provide deeper insight into the fluid dynamics of rotating membrane oxygenators and bioreactors [68].

\section{NANO-BIOCONVECTION TRANSPORT MODEL FORMULATION}

Consider viscous incompressible bioconvective nanofluid swirling flow from an impermeable rotating disk adjacent to a saturated Darcy porous medium. Anisotropic slip and Stefan blowing effects are included at the disk surface. The disk is stretched radially with a power-law velocity, of exponent, $m$. The nanofluid is assumed to be in thermal equilibrium. The disk rotates at constant angular velocity $\Omega$ at $\bar{z}=0 \cdot(\bar{u}, \bar{v}, \bar{w})$ are the velocity components along the directions of the cylindrical coordinates $(\bar{r}, \theta, \bar{z})$. The governing equations i.e. balance of mass, momentum, energy, nanoparticle volume fraction and density of motile microorganisms in cylindrical coordinates, following Shevchuk [26] and Chen et al. [69] are given by:

$$
\begin{aligned}
& \frac{1}{\bar{r}} \frac{\partial}{\partial \bar{r}}(\overline{r u})+\frac{\partial \bar{w}}{\partial \bar{z}}=0 \\
& \bar{u} \frac{\partial \bar{u}}{\partial \bar{r}}+\bar{w} \frac{\partial \bar{u}}{\partial \bar{z}}-\frac{\bar{v}^{2}}{\bar{r}}=v\left(\frac{\partial^{2} \bar{u}}{\partial \bar{r}^{2}}+\frac{1}{\bar{r}} \frac{\partial \bar{u}}{\partial \bar{r}}+\frac{\partial^{2} \bar{u}}{\partial \bar{z}^{2}}-\frac{\bar{u}}{\bar{r}^{2}}\right)-\frac{v}{K\left(\frac{\bar{r}}{R}\right)} \bar{u} \\
& \bar{u} \frac{\partial \bar{v}}{\partial \bar{r}}+\bar{w} \frac{\partial \bar{v}}{\partial \bar{z}}+\frac{\overline{u v}}{\bar{r}}=v\left(\frac{\partial^{2} \bar{v}}{\partial \bar{r}^{2}}+\frac{1}{\bar{r}} \frac{\partial \bar{v}}{\partial \bar{r}}+\frac{\partial^{2} \bar{v}}{\partial \bar{z}^{2}}-\frac{\bar{v}}{\bar{r}^{2}}\right)-\frac{v}{K\left(\frac{\bar{r}}{\bar{R}}\right)} \bar{v} \\
& \bar{u} \frac{\partial \bar{w}}{\partial \bar{r}}+\bar{w} \frac{\partial \bar{w}}{\partial \bar{z}}=v\left(\frac{\partial^{2} \bar{w}}{\partial \bar{r}^{2}}+\frac{1}{\bar{r}} \frac{\partial \bar{w}}{\partial \bar{r}}+\frac{\partial^{2} \bar{w}}{\partial \bar{z}^{2}}\right) \\
& \bar{u} \frac{\partial \bar{T}}{\partial \bar{r}}+\bar{w} \frac{\partial \bar{T}}{\partial \bar{z}}=\alpha\left(\frac{\partial^{2} \bar{T}}{\partial \bar{r}^{2}}+\frac{1}{\bar{r}} \frac{\partial \bar{T}}{\partial \bar{r}}+\frac{\partial^{2} \bar{T}}{\partial \bar{z}^{2}}\right)+\tau D_{B}\left(\frac{\partial \bar{C}}{\partial \bar{r}} \frac{\partial \bar{T}}{\partial \bar{r}}+\frac{\partial \bar{C}}{\partial \bar{z}} \frac{\partial \bar{T}}{\partial \bar{z}}\right)+\frac{\tau D_{T}}{T_{\infty}}\left\{\left(\frac{\partial \bar{T}}{\partial \bar{r}}\right)^{2}+\left(\frac{\partial \bar{T}}{\partial \bar{z}}\right)^{2}\right\} \\
& \bar{u} \frac{\partial \bar{C}}{\partial \bar{r}}+\bar{w} \frac{\partial \bar{C}}{\partial \bar{z}}=D_{B}\left(\frac{\partial^{2} \bar{C}}{\partial \bar{r}^{2}}+\frac{1}{\bar{r}} \frac{\partial \bar{C}}{\partial \bar{r}}+\frac{\partial^{2} \bar{C}}{\partial \bar{z}^{2}}\right)+\frac{D_{T}}{T_{\infty}}\left(\frac{\partial^{2} \bar{T}}{\partial \bar{r}^{2}}+\frac{1}{\bar{r}} \frac{\partial \bar{T}}{\partial \bar{r}}+\frac{\partial^{2} \bar{T}}{\partial \bar{z}^{2}}\right) \\
& \bar{u} \frac{\partial \bar{n}}{\partial \bar{r}}+\bar{w} \frac{\partial \bar{n}}{\partial \bar{z}}+\frac{\partial}{\partial \bar{z}}(\bar{n} \tilde{v})=D_{n}\left(\frac{\partial^{2} \bar{n}}{\partial \bar{r}^{2}}+\frac{1}{\bar{r}} \frac{\partial \bar{n}}{\partial \bar{r}}+\frac{\partial^{2} \bar{n}}{\partial \bar{z}^{2}}\right) .
\end{aligned}
$$

The appropriate boundary conditions are (Fang [70], Lienhard and Lienhard [47):

At $\bar{z}=0$ :

$$
\begin{aligned}
& \bar{u}=N_{1}\left(\frac{\bar{r}}{R}\right) v \frac{\partial \bar{u}}{\partial \bar{z}}, \quad \bar{v}=N_{2}\left(\frac{\bar{r}}{R}\right) v \frac{\partial \bar{v}}{\partial \bar{z}}+\left(\frac{\bar{r}}{R}\right)^{1-2 m} \Omega R, \quad \bar{w}=\frac{-D_{B}}{1-C_{w}} \frac{\partial \bar{C}}{\partial \bar{z}}, \\
& \bar{T}=T_{w}+D_{1}\left(\frac{\bar{r}}{R}\right) \frac{\partial \bar{T}}{\partial \bar{z}}, \quad \bar{C}=C_{w}+E_{1}\left(\frac{\bar{r}}{R}\right) \frac{\partial \bar{C}}{\partial \bar{z}}, \quad \bar{n}=n_{w}+F_{1}\left(\frac{\bar{r}}{R}\right) \frac{\partial \bar{n}}{\partial \bar{z}},
\end{aligned}
$$

The dimensional quantities are: $T$ : temperature, $n:$ number of motile micro-organisms, $C$ : nanoparticle concentration, $\rho$ : fluid density, $v$ : kinematic coefficient of viscosity, $K\left(=K_{0} r^{2 m}\right)$ : variable permeability, $K_{0}$ : constant permeability, $R$ : reference scale length, $\alpha$ : thermal diffusivity $(\rho c)_{f}$ : fluid heat capacity, $D_{B}$ : Brownian diffusion coefficient, $(\rho c)_{p}$ : effective nanoparticles heat capacity, $D_{n}$ : microorganism diffusion coefficient, $D_{T}$ : thermophoretic diffusion coefficient, $T_{\infty}$ : ambient temperature, $T_{w}$ : surface temperature, $C_{\infty}$ : ambient mass concentration, $C_{w}$ : surface mass 
concentration, $N_{2}\left(=\left(N_{2}\right)_{0} r^{m}\right)$ : velocity slip factor along $\bar{v}, N_{1}\left(=\left(N_{1}\right)_{0} r^{m}\right)$ : velocity slip factor along $\bar{u}, D_{1}\left(=\left(D_{1}\right)_{0} r^{m}\right)$ : thermal slip factor, $E_{1}\left(=\left(E_{1}\right)_{0} r^{m}\right)$ : mass slip factor, $n_{w}$ : wall motile microorganisms, $F_{1}\left(=\left(F_{1}\right)_{0} r^{m}\right):$ microorganism slip factor, $m$ : power law exponent, $\tilde{v}\left(=\frac{\tilde{b} W_{c}}{\Delta C} \frac{\partial \bar{C}}{\partial \bar{z}}\right)$ : average directional swimming velocity of microorganisms, $\tilde{b}$ : chemotaxis constant, $W_{c}$ : maximum cell swimming speed. The dimensionless parameters are ${ }_{\tau}$ : ratio of effective nanoparticle heat capacity to thd fluid heat capacity, and $m$ : power law exponent. Proceeding with the analysis the following dimensionless variables are introduced:

$$
r=\frac{\bar{r}}{R}, z=\frac{\bar{z}}{R} \sqrt{\operatorname{Re}}, u=\frac{\bar{u}}{R \Omega}, v=\frac{\bar{v}}{\Omega R}, w=\frac{\bar{w} \sqrt{\mathrm{Re}}}{\Omega R}, \chi=\frac{\bar{n}}{\bar{n}_{w}}, \theta=\frac{\bar{T}-T_{\infty}}{T_{w}-T_{\infty}}, \phi=\frac{\bar{C}-C_{\infty}}{C_{w}-C_{\infty}} .
$$

The dimensionless form of the conservations equations are therefore:

$\frac{\partial u}{\partial r}+\frac{u}{r}+\frac{\partial w}{\partial z}=0$

$u \frac{\partial u}{\partial r}+w \frac{\partial u}{\partial z}-\frac{v^{2}}{r}=\frac{1}{\operatorname{Re}}\left(\frac{\partial^{2} u}{\partial r^{2}}+\frac{1}{r} \frac{\partial u}{\partial r}-\frac{u}{r^{2}}\right)+\frac{\partial^{2} u}{\partial z^{2}}-\frac{\mu}{\Omega \rho K_{0} r^{2 m}} u$,

$u \frac{\partial v}{\partial r}+w \frac{\partial v}{\partial z}+\frac{u v}{r}=\frac{1}{\operatorname{Re}}\left(\frac{\partial^{2} v}{\partial r^{2}}+\frac{1}{r} \frac{\partial v}{\partial r}-\frac{v}{r^{2}}\right)+\frac{\partial^{2} v}{\partial z^{2}}-\frac{\mu}{\Omega \rho K_{0} r^{2 m}} v$

$\frac{1}{\sqrt{\operatorname{Re}}}\left(u \frac{\partial w}{\partial r}+w \frac{\partial w}{\partial z}\right)=\frac{1}{\operatorname{Re}^{3 / 2}}\left(\frac{\partial^{2} w}{\partial r^{2}}+\frac{1}{r} \frac{\partial w}{\partial r}\right)+\frac{1}{\sqrt{\operatorname{Re}}} \frac{\partial^{2} w}{\partial r^{2}}$,

$u \frac{\partial \theta}{\partial r}+w \frac{\partial \theta}{\partial z}=\frac{1}{R \mathrm{e} \operatorname{Pr}}\left\{\frac{\partial^{2} \theta}{\partial r^{2}}+\frac{1}{r} \frac{\partial \theta}{\partial r}+\frac{N b}{\operatorname{Pr}} \frac{\partial \theta}{\partial r} \frac{\partial \phi}{\partial r}+\frac{N t}{\operatorname{Pr}}\left(\frac{\partial \theta}{\partial r}\right)^{2}\right\}$

$+\frac{1}{\operatorname{Pr}}\left\{\frac{\partial^{2} \theta}{\partial z^{2}}+N b\left(\frac{\partial \theta}{\partial z} \frac{\partial \phi}{\partial z}\right)+N t\left(\frac{\partial \theta}{\partial z}\right)^{2}\right\}$,

$u \frac{\partial \phi}{\partial r}+w \frac{\partial \phi}{\partial z}=\frac{1}{L e \operatorname{RePr}}\left\{\frac{\partial^{2} \phi}{\partial r^{2}}+\frac{1}{r} \frac{\partial \phi}{\partial r}+\frac{N t}{N b}\left(\frac{\partial^{2} \theta}{\partial r^{2}}+\frac{1}{r} \frac{\partial \theta}{\partial r}\right)\right\}+\frac{1}{L e \operatorname{Pr}}\left(\frac{\partial^{2} \phi}{\partial z^{2}}+\frac{N t}{N b} \frac{\partial^{2} \theta}{\partial z^{2}}\right)$,

$u \frac{\partial \chi}{\partial r}+w \frac{\partial \chi}{\partial z}=\frac{1}{S b} \frac{\partial^{2} \chi}{\partial z^{2}}-\frac{P e}{S b}\left(\frac{\partial \chi}{\partial z} \frac{\partial \phi}{\partial z}+\chi \frac{\partial^{2} \phi}{\partial z^{2}}\right)$

The boundary conditions now become:

At $z=0$ :

$$
\begin{aligned}
& u=N_{1}(r) \frac{v \sqrt{\operatorname{Re}}}{R} \frac{\partial u}{\partial z}, \quad v=N_{2}(r) \frac{v \sqrt{\operatorname{Re}}}{R} \frac{\partial v}{\partial z}+r^{1-2 m}, \quad w=\frac{-s}{L e P \mathrm{r}} \frac{\partial \phi}{\partial z} \\
& \theta=D_{1}(r) \frac{\sqrt{\operatorname{Re}}}{R} \frac{\partial \theta}{\partial z}+1, \quad \phi=E_{1}(r) \frac{\sqrt{\operatorname{Re}}}{R} \frac{\partial \phi}{\partial z}+1, \quad \theta=F_{1}(r) \frac{\sqrt{\operatorname{Re}} \frac{\partial \chi}{R}}{\partial z}+1
\end{aligned}
$$

As $z \rightarrow \infty:$

$u \rightarrow 0, \quad v \rightarrow 0, \quad \theta \rightarrow 0, \quad \phi \rightarrow 0, \quad \chi \rightarrow 0$.

Invoking the following invariant coordinate transformations based on group theoretical methods, following Hansen [71]: 
$\eta=z r^{-m}, u=r^{1-2 m} f^{\prime}(\eta), v=r^{1-2 m} g(\eta), w=-r^{-m}\left[(2-m) f(\eta)-m \eta f^{\prime}(\eta)\right]$,

$\theta=\theta(\eta), \phi=\phi(\eta), \chi=\chi(\eta)$.

Using (20), equations (12)-(17) may be rendered into the following similarity ordinary differential equations (SODEs)

$$
\begin{aligned}
& f^{\prime \prime \prime}+(2-m) f^{\prime \prime} f-(1-2 m)\left(f^{\prime}\right)^{2}+g^{2}-\frac{1}{D a} f^{\prime}=0, \\
& g^{\prime \prime}+(2-m) f g^{\prime}-(2-2 m) f^{\prime} g-\frac{1}{D a} g=0, \\
& \theta^{\prime \prime}+\operatorname{Pr}(2-m) f \theta^{\prime}+N b \theta^{\prime} \phi^{\prime}+N t\left(\theta^{\prime}\right)^{2}=0, \\
& \phi^{\prime \prime}+\operatorname{Le} \operatorname{Pr}(2-m) f \phi^{\prime}+\frac{N t}{N b} \theta^{\prime \prime}=0, \\
& \chi^{\prime \prime}+\operatorname{Sb}(2-m) f \chi^{\prime}-\operatorname{Pe}\left(\chi^{\prime} \phi^{\prime}+\chi \phi^{\prime \prime}\right)=0 .
\end{aligned}
$$

The boundary conditions (18), (19) assume the form:

$$
\begin{aligned}
& f^{\prime}(0)=\delta_{u} f^{\prime \prime}(0), g(0)=1+\delta_{v} g^{\prime}(0), \quad f(0)=\frac{s}{L e \operatorname{Pr}(2-m)} \phi^{\prime}(0), \\
& \theta(0)=1+\delta_{T} \theta^{\prime}(0), \quad \phi(0)=1+\delta_{C} \phi^{\prime}(0), \quad \chi(0)=1+\delta_{n} \chi^{\prime}(0), \\
& f^{\prime}(+\infty) \rightarrow 0, \quad g(+\infty) \rightarrow 0, \quad \theta(+\infty) \rightarrow 0, \quad \phi(+\infty) \rightarrow 0, \quad \chi(+\infty) \rightarrow 0 .
\end{aligned}
$$

The dimensionless parameters arising are as follows: $f$ :axial stream function, $g$ : circumferential stream function, Re: rotational Reynolds number, $m$ : power-law stretching rate of disk, $D a$ : Darcy number, $\theta$ : temperature, $\phi:$ nanoparticles volume fraction, $N b$ : Brownian motion, $\operatorname{Pr}$ : Prandtl number, $L e$ : Lewis number, $N t$ : thermophoresis, $\chi$ : density of motile microorganism, $S b$ : bioconvection Schmidt number, $P e$ : bioconvection Péclet number, $\delta_{v}$ : circumferential slip, $\delta_{u}$ : radial slip, $\delta_{C}:$ mass slip, $\delta_{T}$ : thermal slip, $\delta n$ : microorganism slip, $s$ : Stefan blowing parameter. The appropriate definitions are:

$$
\begin{aligned}
& \operatorname{Re}=\frac{\Omega R^{2}}{v}, D a^{-1}=\frac{v}{\Omega K_{0}}, \operatorname{Pr}=\frac{v}{\alpha}, N b=\frac{\tau D_{B} \Delta C}{\alpha}, N t=\frac{\tau D_{T} \Delta T}{\alpha T_{\infty}}, L e=\frac{\alpha}{D_{B}}, P e=\frac{\tilde{b} W c}{D_{B}}, s=\frac{\Delta C}{1-C_{w}}, \\
& S b=\frac{v}{D_{n}}, \delta_{u}=\frac{\left(N_{1}\right)_{0} v \sqrt{\operatorname{Re}}}{R}, \delta_{v}=\frac{\left(N_{2}\right)_{0} v \sqrt{\operatorname{Re}}}{R}, \delta_{T}=\frac{\left(D_{1}\right)_{0} \sqrt{\operatorname{Re}}}{R}, \delta_{C}=\frac{\left(E_{1}\right)_{0} \sqrt{\operatorname{Re}}}{R}, \delta_{n}=\frac{\left(F_{1}\right)_{0} \sqrt{\operatorname{Re}}}{R} .
\end{aligned}
$$

Here, $s>0$ represents mass flux moving out from the disk to the free stream while $s<0$ is the mass flux moving into the disk, following the convention of Fang [70].

\section{DISK SURFACE VARIABLE RATES}

The important physical design quantities in engineering e.g. rotating disk membrane oxygenators, relate to the gradients of key variables at the disk surface (wall) i.e. momentum, heat, nanoparticle mass and micro-organism transfer. These are respectively the local skin friction in the $\bar{r}$-direction: $C_{f \bar{r}}$, local skin friction in $\theta$-direction $C_{f \theta}$, local Sherwood number $s h_{\bar{r}}$, local wall motile microorganism number density gradient $N n_{\bar{r}}$, and local Nusselt number $N u_{\bar{r}}$ which are defined as: 
$C_{f \bar{r}}=\frac{\tau_{\bar{r}}}{\rho_{f} \bar{u}^{2}}, C_{f \theta}=\frac{\tau_{\theta}}{\rho_{f} \bar{v}^{-2}}, N u_{\bar{r}}=\frac{\bar{r} q_{w}}{k_{f}\left(T_{w}-T_{\infty}\right)}$,

$S h_{\bar{r}}=\frac{\bar{r} q_{m}}{D_{B}\left(C_{w}-C_{\infty}\right)}, N n_{\bar{r}}=\frac{\bar{r} q_{n}}{D_{n} n_{w}}$,

Here $\tau_{\bar{r}}, \tau_{\theta}, q_{w}, q_{m}$ and $q_{n}$ denote the shear stress in $\bar{r}$-direction, shear stress in $\theta$-direction, surface mass flux, surface heat flux, and the surface motile micro-organism flux respectively. The terms are given as follows:

$$
\begin{aligned}
& \tau_{\bar{r}}=\mu\left[\frac{\partial \bar{u}}{\partial \bar{z}}+\frac{\partial \bar{w}}{\partial \bar{r}}\right]_{\bar{z}=0}, \tau_{\theta}=\mu\left[\frac{\partial \bar{v}}{\partial \bar{z}}+\frac{\partial \bar{w}}{\partial \bar{r}}\right]_{\bar{z}=0}, q_{w}=-k\left[\frac{\partial T}{\partial \bar{z}}\right]_{\bar{z}=0}, \\
& q_{m}=-D_{B}\left[\frac{\partial C}{\partial \bar{z}}\right]_{\bar{z}=0}, q_{n}=-D_{n}\left[\frac{\partial n}{\partial \bar{z}}\right]_{\bar{z}=0} .
\end{aligned}
$$

Using Eqns. (20), (30) in Eqn. (29), we have:

$\operatorname{Re}_{\bar{r}}^{1 / 2} C_{f \bar{r}}=f^{\prime \prime}(0), \quad \operatorname{Re}_{\bar{r}}^{1 / 2} C_{f \theta}=g^{\prime}(0), \quad \operatorname{Re}_{\bar{r}}^{-1 / 2} N u_{\bar{r}}=-\theta^{\prime}(0)$,

$\operatorname{Re}_{\bar{r}}^{-1 / 2} S h_{\bar{r}}=-\phi^{\prime}(0), \quad \operatorname{Re}_{\bar{r}}^{-1 / 2} N n_{\bar{r}}=-\chi^{\prime}(0)$,

where $\operatorname{Re}_{\bar{r}}=\frac{\Omega R^{2}}{v} r^{-2(m-1)}$ denotes the local rotational Reynolds number.

\section{COMPUTATIONAL SOLUTIONS WITH CHEBYCHEV COLLOCATION}

As noted earlier, the Chebyshev collocation method is very accurate and achieves exceptional convergence. An excellent appraisal of this method is given in Canuto et al. [55]. The advantage of this method over traditional finite element method is the accuracy of this method with lower number of discretized points, since the method uses compact matrix to represent the polynomial. Furthermore, the method can handle the non-periodic boundary conditions, while its competitor, Fast Fourier Transform method cannot directly handle non-periodic boundary conditions.

To implement this method, the similarity ordinary differential equations (SODEs) are initially transformed into a set of second order SODEs as follows: Assume that $F_{1}=f, F_{2}=f^{\prime}, F_{3}=g, F_{4}=$ $\theta, F_{5}=\phi, F_{6}=\chi$. With the introduction of the new variables, the SODEs are expressed in terms of the following set of SODEs of second order: 


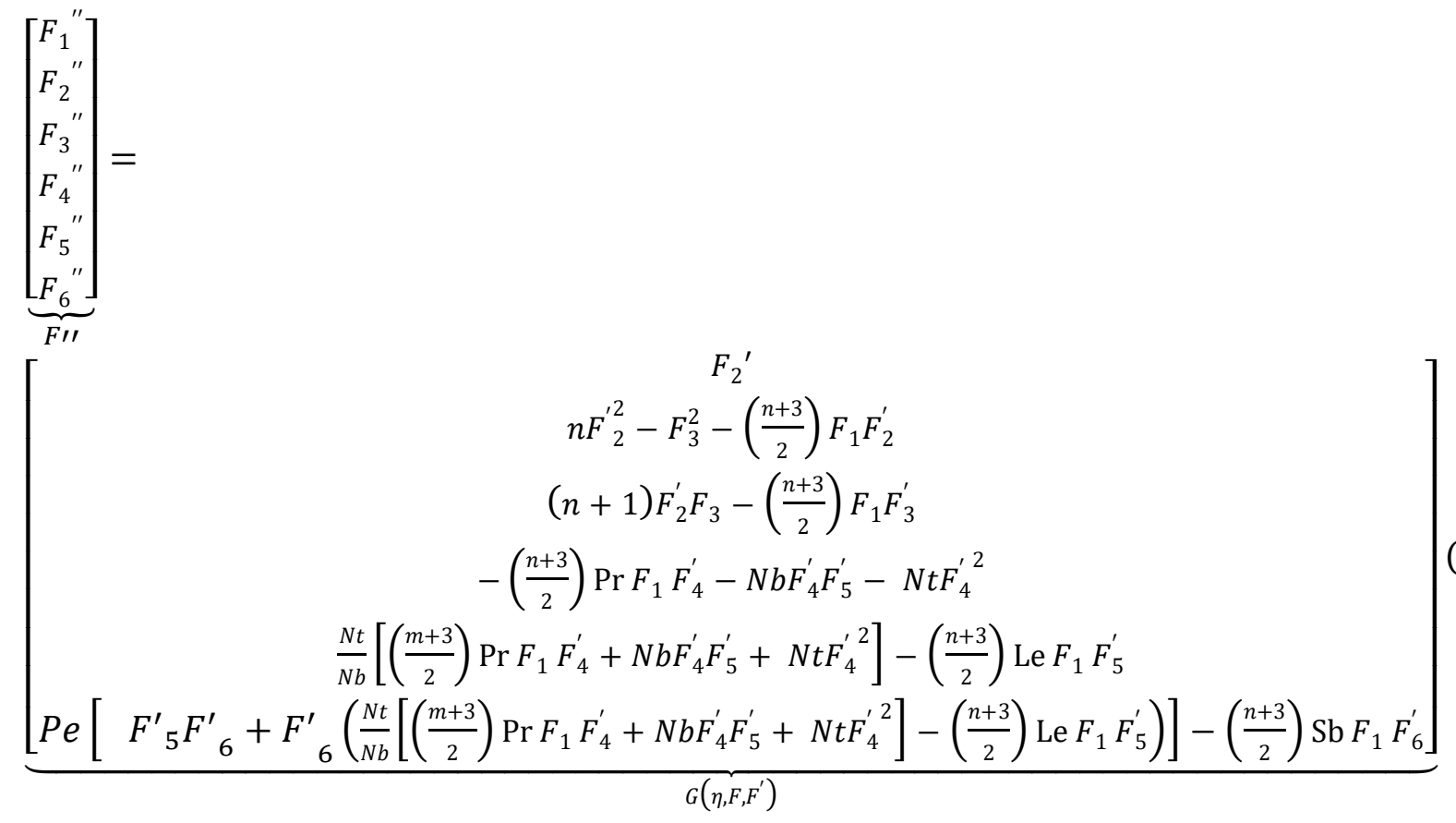

Thus, it follows that $F^{\prime \prime}$ is a function of $\eta, F:=\left[F_{1}, F_{2}, F_{3}, F_{4}, F_{5}, F_{6}\right]^{T}$ and $F^{\prime}$.

Furthermore, the boundary conditions in Eqns. (26) - (27) can be presented as:

$$
\begin{gathered}
F_{1}(0)=0 \\
F_{2}(0)=0 \\
F_{3}(0)=1 \\
F_{4}(0)=1 \\
F_{5}(0)=1 \\
F_{2}(\infty)=0 \\
F_{3}(\infty)=0 \\
F_{4}(\infty)=0 \\
F_{5}(\infty)=0
\end{gathered}
$$

The domain of SODEs is discretized with $n$ Chebyshev collocation points $x_{j}$ in the Chebyshev domain $[-1,1]$ with $j=1,2, \ldots, n$. The $j$-th Chebyshev collocation point is determined by $x_{j}=\cos (j \pi / n)$ The derivative of $F$ can be deduced by Chebyshev derivative $D F$ where $D$ denotes an $(n+1) \times(n+1)$ matrix of the form:

$$
\left.\begin{array}{c}
D_{00}=\frac{2 n^{2}+1}{6} \\
D_{n n}=-\frac{2 n^{2}+1}{6} \\
D_{j j}=\frac{-x_{j}}{2\left(1-x_{j}^{2}\right)} \quad \text { for } j=1, \ldots, n-1 \\
=\left(\frac{c_{i}}{c_{j}}\right) \frac{(-1)^{i+j}}{\left(x_{i}-x_{j}\right)} \text { for } \mathrm{i} \neq j, i, j=1, \ldots, n-1
\end{array}\right\} c_{i}=\left\{\begin{array}{cc}
2 & i=0 \text { or } n \\
1 & \text { otherwise }
\end{array}\right\}
$$

The second derivative of the model variables $F$ can be approximated by $D^{2} F$ where $D^{2}=D \times D$. Utilizing Chebyshev collocation on Eqn. (32), a system of $6(n+1)$ non-linear equations is formulated. Furthermore, we note that the physical range of $\eta$ is given by $[0, \infty]$. However, from the actual simulations, it is apparent that the upper bound of $\eta=10$ sufficiently represents the upper bound $\eta=$ $\infty$. Therefore, we require to map the Chebyshev collocation domain [-1 1] to the physical domain $[0$, $10]$ where the mapping can be derived as:

$x=\frac{\eta-5}{5}$.

Eqn. (32) can be expressed in the Chebyshev domain as: 
$\underbrace{\left[\begin{array}{c}D^{2} F_{1} \\ D^{2} F_{1} \\ D^{2} F_{1} \\ D^{2} F_{1} \\ D^{2} F_{1} \\ D^{2} F_{1}\end{array}\right]}_{F \prime \prime}=$

$\left[\begin{array}{c}D F_{2} \\ n D F_{2}^{2}-F_{3}^{2}-\left(\frac{n+3}{2}\right) F_{1} D F_{2} \\ (n+1) D F_{2} F_{3}-\left(\frac{n+3}{2}\right) F_{1} D F_{3} \\ -\left(\frac{n+3}{2}\right) \operatorname{Pr} F_{1} D F_{4}-N b D F_{4} D F_{5}-N t D F_{4}{ }^{2} \\ \operatorname{Pe}\left[D F_{5} D F_{6}+D F_{6}\left(\frac{N t}{N b}\left[\left(\frac{m+3}{2}\right) \operatorname{Pr} F_{1} D F_{4}+N b D F_{4} D F_{5}+N t D F_{4}{ }^{2}\right]-\left(\frac{n+3}{2}\right) \operatorname{Le} F_{1} D F_{5}\right)\right]-\left(\frac{n+3}{2}\right) \operatorname{Sb} F_{1} D F_{6}\end{array}\right]$

The boundary conditions remain the same as in Eqn. (33). As an example, the first equation of the matrix-vector Eq. (36) is derived as:

$D^{2} F_{1}=\left[\begin{array}{cccc}D_{00}^{2} & D_{01}^{2} & \cdots & D_{0 n}^{2} \\ D_{10}^{2} & D_{11}^{2} & \cdots & D_{1 n}^{2} \\ \vdots & \vdots & \vdots & \vdots \\ D_{n 0}^{2} & D_{n 1}^{2} & \cdots & D_{n n}^{2}\end{array}\right]\left[\begin{array}{c}F_{1,0} \\ F_{1,1} \\ \vdots \\ F_{1, n}\end{array}\right]=\left[\begin{array}{cccc}D_{00} & D_{01} & \cdots & D_{0 n} \\ D_{10} & D_{11} & \cdots & D_{1 n} \\ \vdots & \vdots & \vdots & \vdots \\ D_{n 0} & D_{n 1} & \cdots & D_{n n}\end{array}\right]\left[\begin{array}{c}F_{2,0} \\ F_{2,1} \\ \vdots \\ F_{2, n}\end{array}\right]$

The equation can be formulated as:

$\left[\begin{array}{cccc}D_{00}^{2} & D_{01}^{2} & \cdots & D_{0 n}^{2} \\ D_{10}^{2} & D_{11}^{2} & \cdots & D_{1 n}^{2} \\ \vdots & \vdots & \vdots & \vdots \\ D_{n 0}^{2} & D_{n 1}^{2} & \cdots & D_{n n}^{2}\end{array}\right]\left[\begin{array}{c}F_{1,0} \\ F_{1,1} \\ \vdots \\ F_{1, n}\end{array}\right]=\left[\begin{array}{c}D_{00} F_{2,0}+D_{01} F_{2,1}+\cdots+D_{0 n} F_{2, n} \\ D_{10} F_{2,0}+D_{11} F_{2,1}+\cdots+D_{1 n} F_{2, n} \\ \vdots \\ D_{n 0} F_{2,0}+D_{n 1} F_{2,1}+\cdots+D_{n n} F_{2, n}\end{array}\right]$

The boundary conditions in Eqn. (36) need to be incorporated into the matrix equations. Boundary conditions are set at the proper locations by replacing the corresponding discretized equations of Eqn. (36). For Eqn. (38), the first equation is substituted by the corresponding boundary equation in Eq. (36):

$\left[\begin{array}{cccr}1 & 0 & \cdots & 0 \\ D_{10}^{2} & D_{11}^{2} & \cdots & D_{1 n}^{2} \\ \vdots & \vdots & \vdots & \vdots \\ D_{(n-1) 0}^{2} & D_{(n-1) 1}^{2} & \cdots & D_{(n-1) n}^{2} \\ D_{n 0} & D_{n 0} & \cdots & D_{n 0}\end{array}\right]\left[\begin{array}{c}F_{1,0} \\ F_{1,1} \\ \vdots \\ F_{1, n-1} \\ F_{1, n}\end{array}\right]=\left[\begin{array}{c}0 \\ D_{10} F_{2,0}+D_{11} F_{2,1}+\cdots+D_{1 n} F_{2, n} \\ \vdots \\ D_{(n-1) 0} F_{2,0}+D_{(n-1) 1} F_{2,1}+\cdots+D_{(n-1) n} F_{2, n} \\ D_{n 0} F_{2,0}+D_{n 1} F_{2,1}+\cdots+D_{n n} F_{2, n}\end{array}\right]$

By the same process, we arrange all the boundary conditions for $D^{2} F_{2}, D^{2} F_{3}, \ldots, D^{2} F_{6}$ in the discretized form of Eqn. (36) which provides a large non-linear system of $6(n+1)$ equations with $6(n+1)$ unknowns. In the current study, using the MATLAB trust-region-reflective algorithm $f$ solve, the non-linear system of equations has been solved. Following this, the solution is re-mapped to the physical domain via the following calculation:

$$
\eta=5 x+5
$$




\section{VALIDATION OF CHEBYSHEV COLLOCATION METHOD}

The nonlinear ordinary boundary value problem defined by Eqns. (21)-(25) with boundary conditions (26)-(27), is of $11^{\text {th }}$ order and strongly coupled. A numerical technique is therefore adopted to solve this system, namely the Chebyshev collocation method. To validate this approach, benchmarking with existing simpler cases from the literature has been conducted. The general model reduces exactly to the model of Chen et al. [69] for the no-slip scenario, i.e., $\delta_{u}=\delta_{v}=\delta_{T}=\delta_{C}=\delta_{n}=0$, purely fluid medium $\left(\frac{1}{D a} \rightarrow 0\right)$ and in the absence of Stefan blowing, $s=0$, along with a minor modification $2 m=1-n$. The comparison is documented in Table $\mathbf{1}$, and generally very close agreement is achieved between the shooting quadrature solutions of Chen et al. [69] and the present collocation computations. Confidence in the present Chebyshev collocation solutions is therefore justifiably high.

Table 1: Computational values of $f^{\prime \prime}(0), h^{\prime}(0),-\theta^{\prime}(0)$ for different values of $n$

\begin{tabular}{|c|c|c|c|c|}
\hline & & $f^{\prime \prime}(0)$ & $g^{\prime}(0)$ & $-\theta^{\prime}(0)$ \\
\hline \multirow[t]{2}{*}{$n=1$} & $\begin{array}{l}\text { Chen et al. }[69] \\
\text { (shooting method) }\end{array}$ & 0.51018 & -0.6149 & 0.4034 \\
\hline & $\begin{array}{l}\text { Present } \\
\text { (Chebyshev } \\
\text { collocation ) }\end{array}$ & 0.50080 & -0.6095 & 0.4028 \\
\hline \multirow[t]{2}{*}{$n=2$} & $\begin{array}{l}\text { Chen et al. }[69] \\
\text { (shooting method) }\end{array}$ & 0.4639 & -0.6677 & 0.4126 \\
\hline & $\begin{array}{l}\text { Present } \\
\text { (Chebyshev } \\
\text { collocation ) }\end{array}$ & 0.4601 & -0.6677 & 0.4073 \\
\hline
\end{tabular}

\section{RESULTS AND DISCUSSION}

An extensive number of computational tests with different parameter values has been conducted. In the simulations, a water-based nanofluid $(P r=6.8)$ is studied and the default parameters are prescribed as follows: $N b=N t=0.1, m=-0.5, D a=10, L e=P e=S b=1$, and $\delta_{\mathrm{c}}=\delta_{\mathrm{n}}=\delta_{\mathrm{T}}=\delta_{\mathrm{u}}=\delta_{\mathrm{v}}=0.1$, unless stated otherwise. Figs. 2-15 illustrate the distributions for the dimensionless variables with transformed coordinate, $\eta$. Inspection of all these plots confirms that $f^{\prime}(\eta) \rightarrow 0, \phi(\eta) \rightarrow 0, \theta(\eta) \rightarrow 0$, and $\chi(\eta) \rightarrow 0$ asymptotically as $\eta \rightarrow \infty$. For the present rotational bioconvective nanofluid flow problem, it is confirmed therefore that $\eta \rightarrow 10$ is large enough to achieve asymptotically smooth solutions at the free stream boundary condition $\eta \rightarrow \infty$. It is worthwhile to state that the accuracy of the solutions for $f^{\prime}(\eta), \phi(\eta), \theta(\eta)$ and $\chi(\eta)$ at $\eta=10$ is achieved of the order $\mathrm{O}\left(10^{-7}\right)$.

Fig. 1 (a-b) and Fig. 2(a-b) illustrate the combined influence of Stefan blowing/suction $(s)$ and Darcy number $D a$ on the dimensionless radial velocity, $f^{\prime}(\eta)$, temperature, $\theta(\eta)$, nano-particle concentration, $\phi(\eta)$ and density of motile micro-organisms, $\chi(\eta)$. It is pertinent to note that suction/injection is not created by holes on the disk surface. The effect of suction (reverse of Stefan blowing) is related to mass flux from the potential flow to the surface of the solid disk. Analogously, the Stefan blowing is produced by the mass flux from the rotating disk surface to the potential flow. As can be seen from Fig. 1(a), radial flow is decreased with strong suction $(s<0)$ whereas the reverse trend is computed for strong blowing $(s>0)$. Evidently the presence of suction inhibits radial momentum diffusion whereas blowing encourages it. The absence of Stefan blowing $(s=0)$ leads to results which naturally fall in between the blowing and suction cases. Although radial deceleration is induced with suction, flow reversal does not arise (radial velocity magnitudes sustain positive values throughout the boundary layer regime transverse to the disk surface). The implication of this result for rotating bioreactor design 
[72] or membrane oxygenator design [73] is that manipulation of mass flux from the potential flow can be employed to control the radial flow field, rather than relying purely on the porosity of the rotating disk, which has been considered in previous designs [74]. This provides the designer with a dual approach to regulate momentum transfer in the near-disk regime and to tune performance, in particular, for membrane oxygenators in neo-natal clinical requirements, as noted by Drummond et al. [75]. Temperature, microorganism and nanoparticle concentration magnitudes in Figs. 1(b) \& 2 (a, b), are all strongly decreased with increasing $D a$. Physically, an increase in the Darcy number reduces the contribution of the Darcian drag force term $-\frac{1}{D a} f^{\prime}$ in the radial momentum Eqn. (21). The impact of increasing Darcy number ( $D a=\frac{\Omega K_{0}}{v}$ ) is to accelerate the radial flow, in particular, in close proximity, to the disk surface (wall). Higher Darcy number implies greater permeability which permits enhanced percolation of the fluid in the porous medium. With the increase of $D a$, temperatures (Fig. 1 (b)) are markedly reduced inside the thermal boundary layer region. The progressive reduction in solid matrix fibers leads to a suppression in thermal conduction heat transfer and this results in a decrease in nanofluid temperatures. It is also observed that a rise in permeability i.e. Darcy number, reduces the concentration level of nanoparticles. Again, the decrease in solid fibers is counter-productive to mass diffusion and depletes the nanoparticle concentration boundary layer thickness. A similar effect is induced in the motile micro-organism diffusion which again is inhibited with greater permeability. However, the reductions in nanoparticle concentration and micro-organism density number are much weaker than those in temperature. The primary influence of Darcy number is on the momentum (radial) since the Darcy body force appears in this equation, not the other conservation equations. Only minor modifications in circumferential (tangential) velocity are computed with large changes in Darcy number, and the plot has been omitted for brevity, even though there is a circumferential Darcy body force in Eqn. (22) i.e. $-g / D a$.

(a)

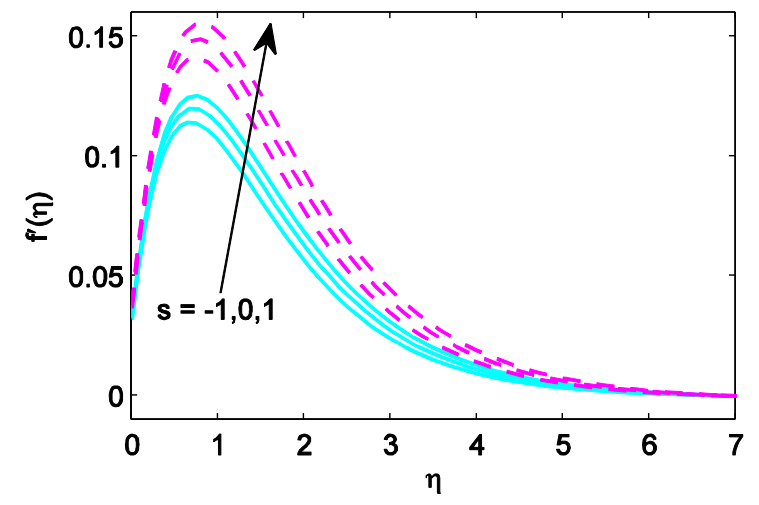

(b)

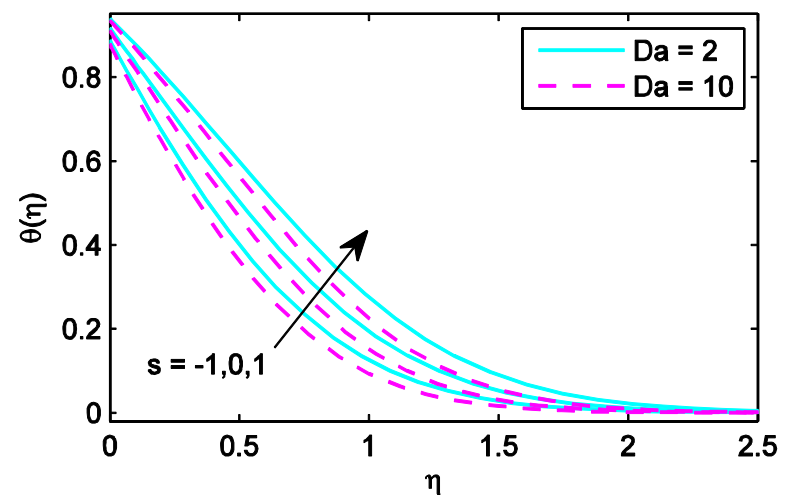

(a)

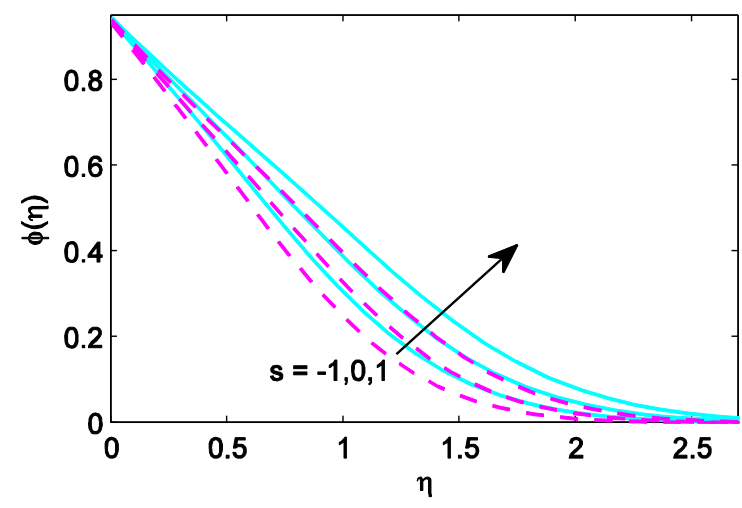

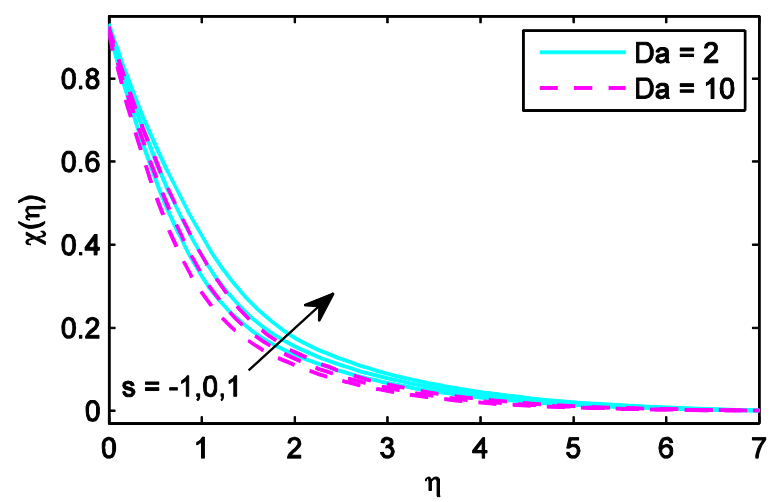

Figure 2: Effects of $s$ and $D a$ on $\phi(\eta)$ and $\chi(\eta)$

Fig. 3 (a)-(b) and Fig. 4 (a)-(b) visualize the collective effect of Stefan blowing/suction $(s)$ and radial slip parameter $\left(\delta_{u}\right)$ on the dimensionless radial velocity, temperature, nano-particle concentration and 
density of micro-organisms. In the vicinity of the wall, radial flow is boosted considerably with greater radial slip parameter $\delta_{u}$ for both cases i.e. with or without the presence of blowing (Fig. 3a). The converse response is computed far away from the wall. This is logical since the primary influence of wall slip is at the disk surface i.e. wall. Any modification in radial momentum here is re-distributed in the free stream, since the Von Karman swirling flow acts similar to a fan drawing fluid in towards the disk surface and thereafter directing it radially outwards towards the disk periphery. Clearly, the incorporation of slip in the present model leads to a deviation from conventional no-slip models, indicating that no-slip models underpredict the radial velocity field and produce erroneous estimates which may be relevant to rotating disk membrane oxygenator efficiency [73]. A higher radial slip value $\delta_{u}$ strongly reduces the temperature, nanoparticle concentration and microorganism density magnitudes (Fig. 3 (b) \& 4 (a, b)) and this is probably attributable to the radial momentum diffusion enhancement exceeding thermal, nanoparticle and microorganism diffusion when radial slip at the disk surface is present.
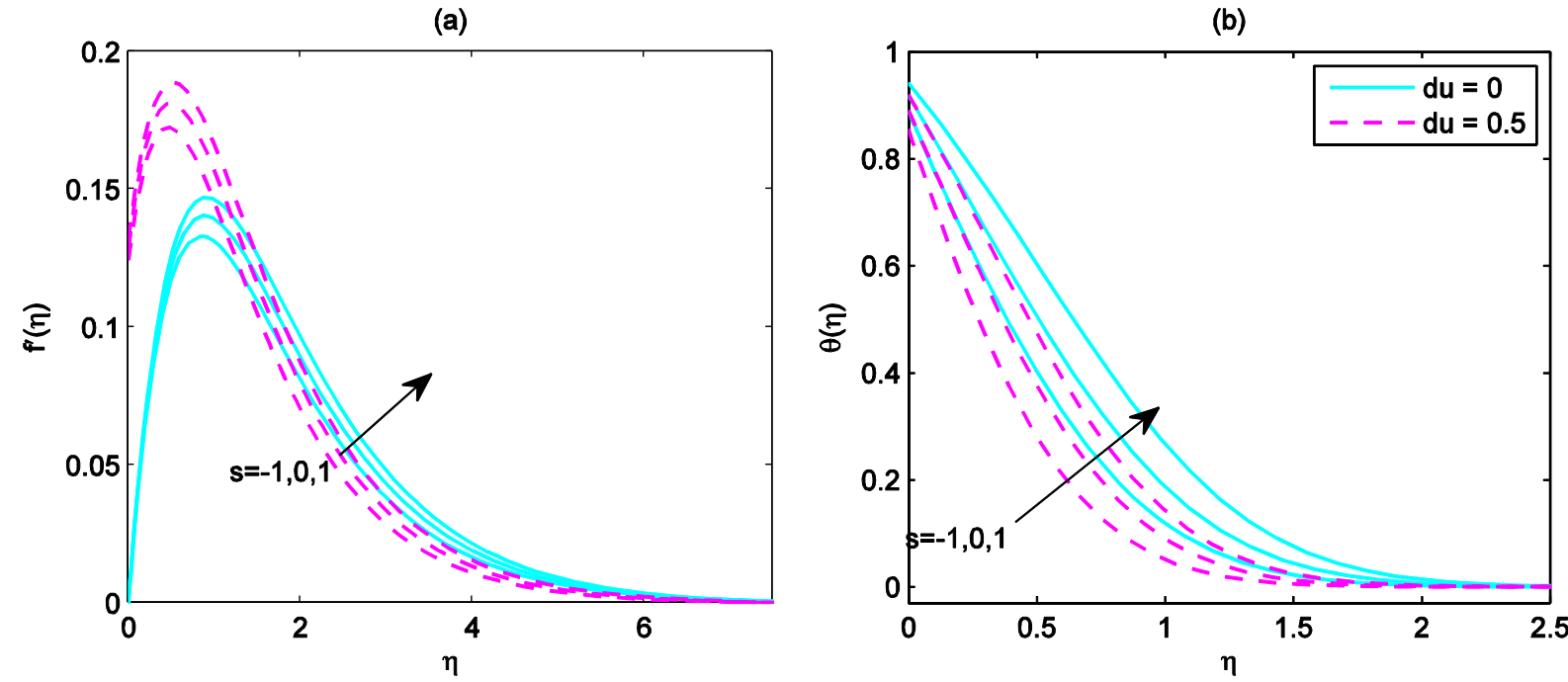

Figure 3: Effects of $s$ and $\delta_{u}$ on $f^{\prime}(\eta)$ and $\theta(\eta)$

(a)

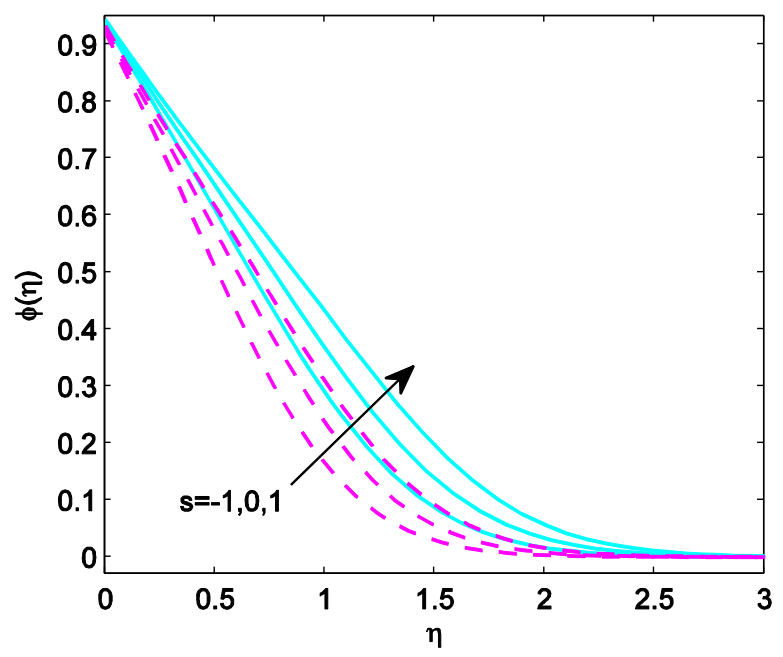

(b)

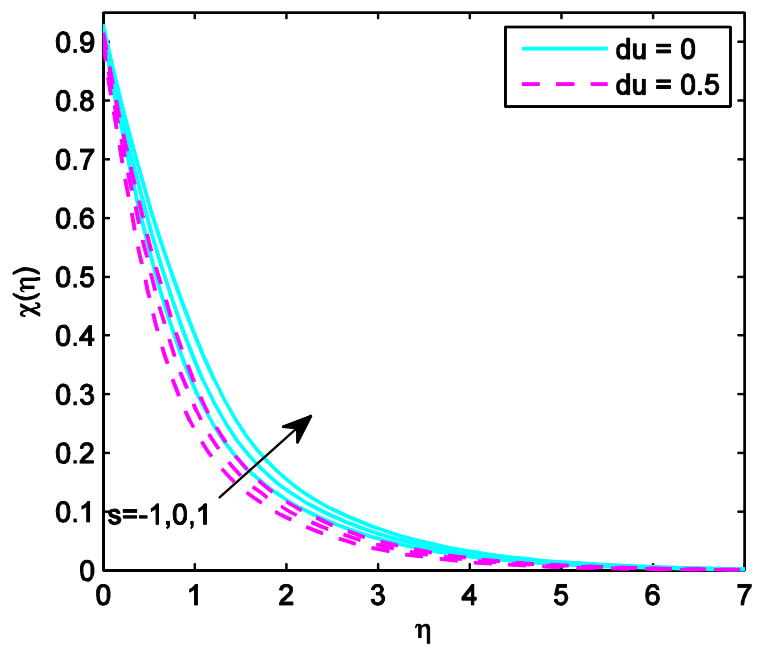

Figure 4: Effects of $s$ and $\delta_{u}$ on $\phi(\eta)$ and $\chi(\eta)$

Figs. 5 and 6 depict the behaviour of circumferential slip parameter $\delta v$ and Stefan blowing parameter, $s$, on the transport characteristics. It is apparent from Fig. 5 (a) that increasing circumferential slip parameter results in a drop in the radial velocity i.e. radial flow deceleration. His is the opposite response to an increase in radial slip (Fig 1a). Evidently there is a re-distribution in momentum in the regime. Since the disk is radially stretched, the primary flow is in the radial direction. Circumferential (tangential) flow is secondary and slip in the circumferential direction will inhibit radial flow. The 
anisotropic nature of the slip imposed therefore exerts different effects on the primary (radial) flow, a feature again of potential relevance to rotating membrane oxygenator designs [74]. Unlike radial slip, the circumferential slip is beneficial to the temperature, concentration and microorganism distributions and generates a significant elevation in these variables which is sustained throughout the boundary layer, as observed in Figs. 5(b), 6(a, b).

(a)

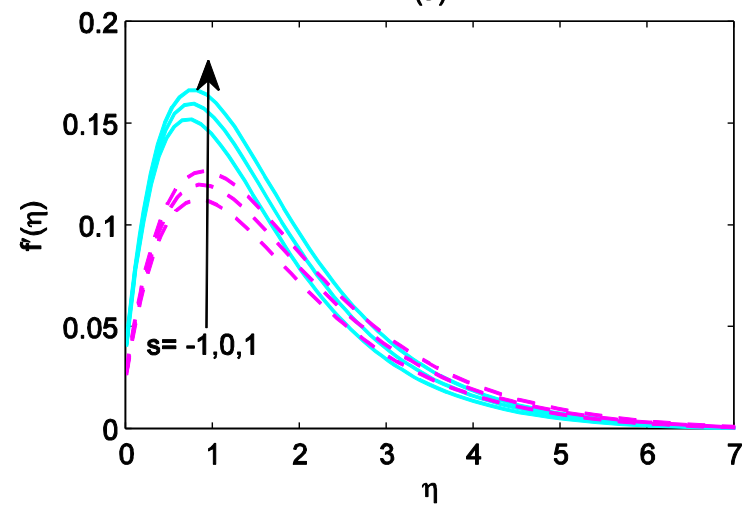

(b)

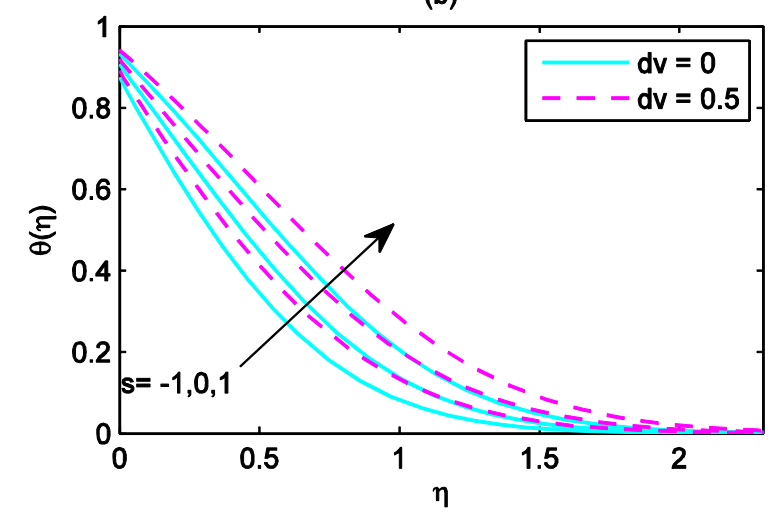

Figure 5: Effects of $s$ and $\delta_{v}$ on $f^{\prime}(\eta)$ and $\theta(\eta)$

(a)

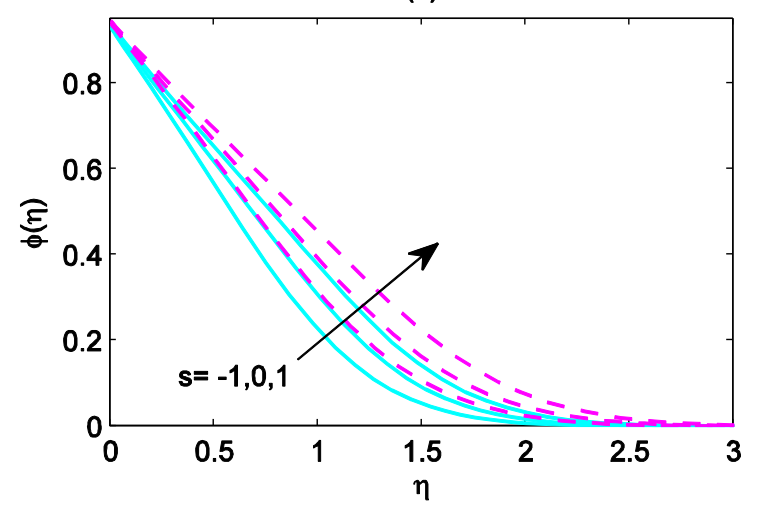

(b)

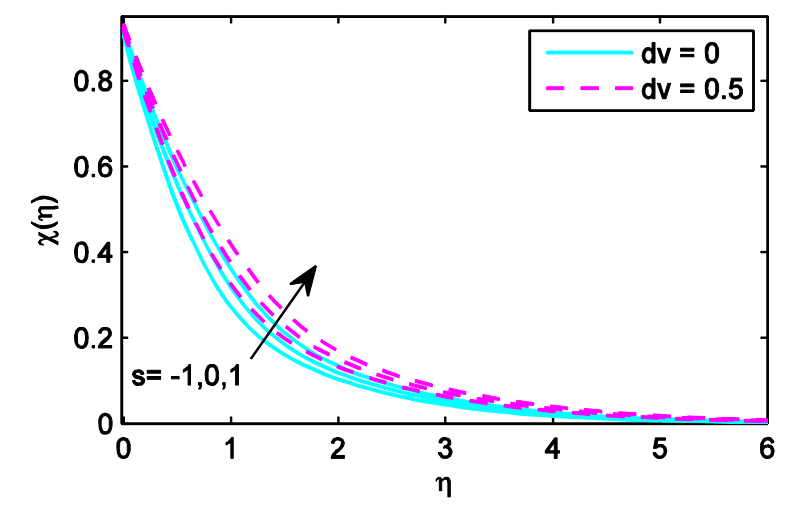

Figure 6: Effects of $s$ and $\delta_{v}$ on $\phi(\eta)$ and $\chi(\eta)$

Figs. 7(a)-(b) and Figs. 8(a)-(b) demonstrates the behaviour of thermal slip parameter $\delta_{T}$ and $s$. Fig. 7(b) shows that a rise in thermal slip parameter leads to a reduction of temperature with or without the presence of blowing. Thermal slip effects on velocity, concentration and microorganism are however not significant. The thermal slip parameter $\delta_{T}$ appears in the boundary condition (26), in the term $\theta(0)=1+\delta_{T} \theta^{\prime}(0)$. Increasing thermal slip leads to a disparity in the temperature ("temperature jump") at the disk surface. Heat transfer from the disk surface to the nanofluid is reduced and this decreases temperatures in the boundary layer and results in a thinner thermal boundary layer.

(a)

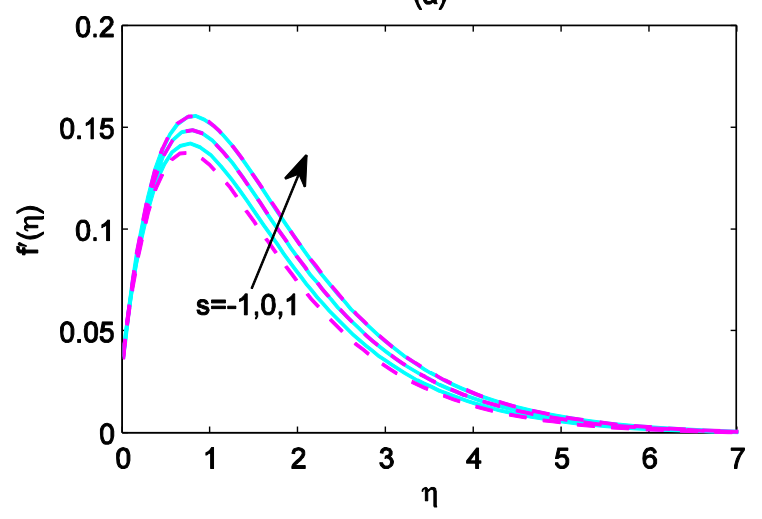

(b)

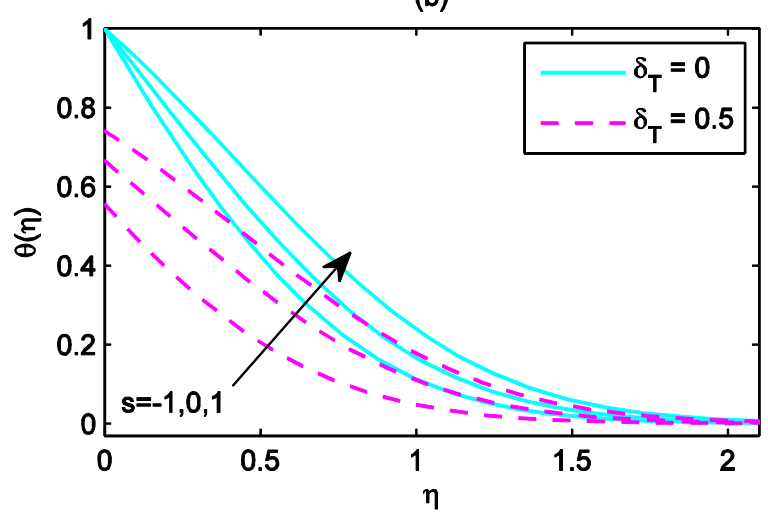

Figure 7: Effects of $s$ and $\delta_{\mathrm{T}}$ on $f^{\prime}(\eta)$ and $\theta(\eta)$ 
(a)

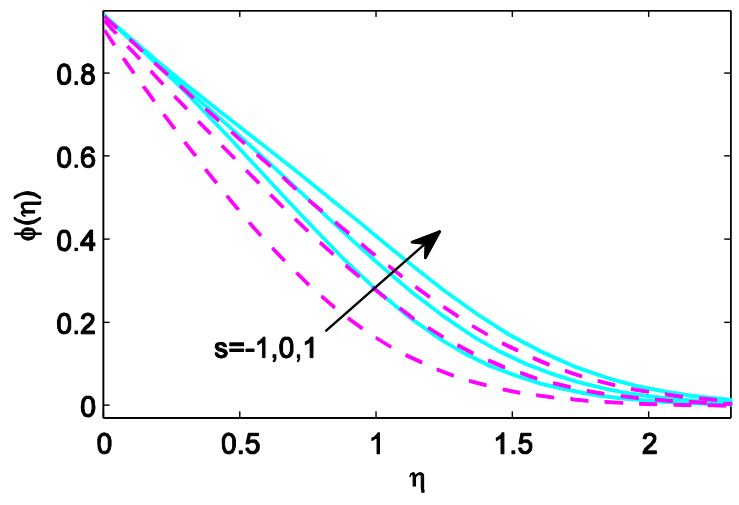

(b)

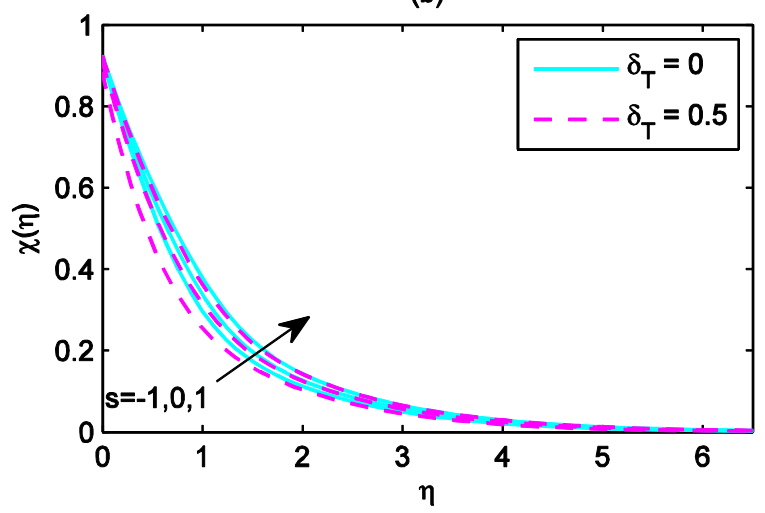

Figure 8: Effects of s and $\delta_{\mathrm{T}}$ on $\phi(\eta)$ and $\chi(\eta)$.

The influence of mass slip parameter $\delta_{C}$ and Stefan blowing parameter, $s$ on the dimensionless radial velocity, temperature, nanoparticle concentration and motile microorganism density number are displayed in Figs. 9 and 10, respectively. It is evident that changing of the sign of parameter $s$ (i.e., from negative to positive) results in an increase in the dimensionless radial velocity, temperature, nanoparticle concentration and motile microorganism density magnitudes, irrespective of the presence or absence of mass slip. Fig. 10(a) shows that a higher mass slip leads to a decline in nanoparticle concentration values. The mass slip parameter $\delta_{C}$ also appears in boundary condition (26), the term $\phi(0)=1+\delta_{C} \phi^{\prime}(0)$. Migration of nanoparticles from the disk surface to the core flow is mitigated with the mass slip effect. There is a more prominent influence at the wall which is progressively reduced into the main body of the nanofluid. Nanoparticle concentrations are therefore over-predicted at the wall when mass slip is absent $\left(\delta_{C}=0\right)$. Increasing mass slip also reduces nanofluid temperature (Fig. $9 \mathrm{~b})$ leading to a depletion in thermal boundary layer thickness. However, there is a weak increase in microorganism density number (Fig. 10b) with increasing nanoparticle mass slip effect. This is probably induced by the strong coupling between the nano-particle concentration and micro-organism density conservation equations via the terms in the latter eqn. (25) i.e. $-P e\left(\chi^{\prime} \phi^{\prime}+\chi \phi^{\prime \prime}\right)$. Although the nanoparticle eqn. (24) and temperature eqn. (23) are coupled, the coupling is weaker than between the nanoparticle and microorganism equations.

(a)

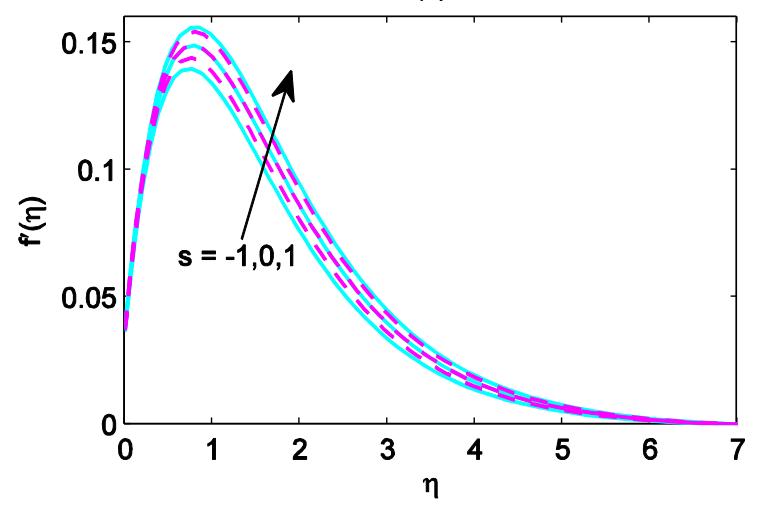

(b)

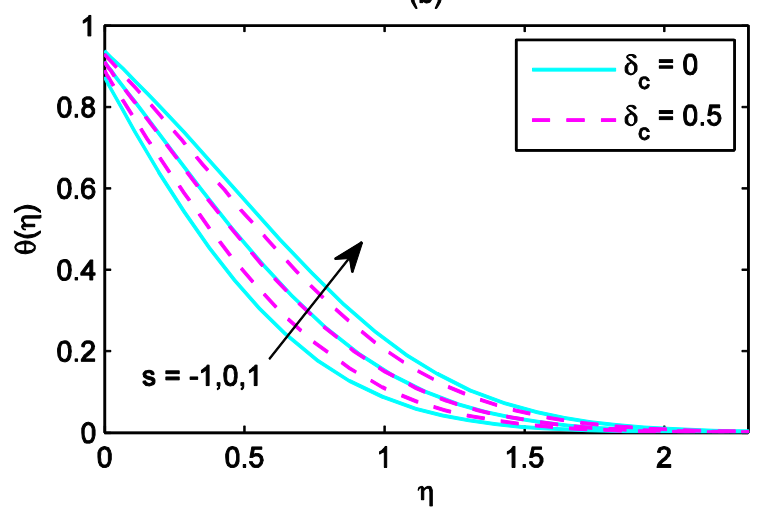

Figure 9: Effects of $s$ and $\delta_{c}$ on $f^{\prime}(\eta)$ and $\theta(\eta)$. 
(a)

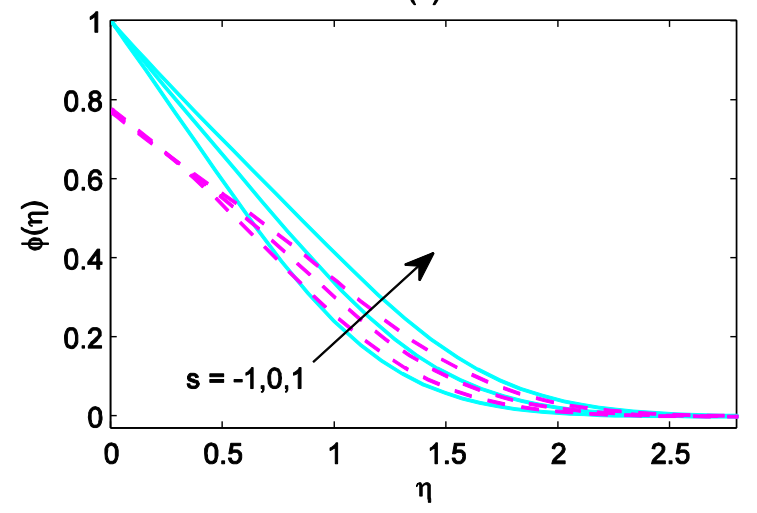

(b)

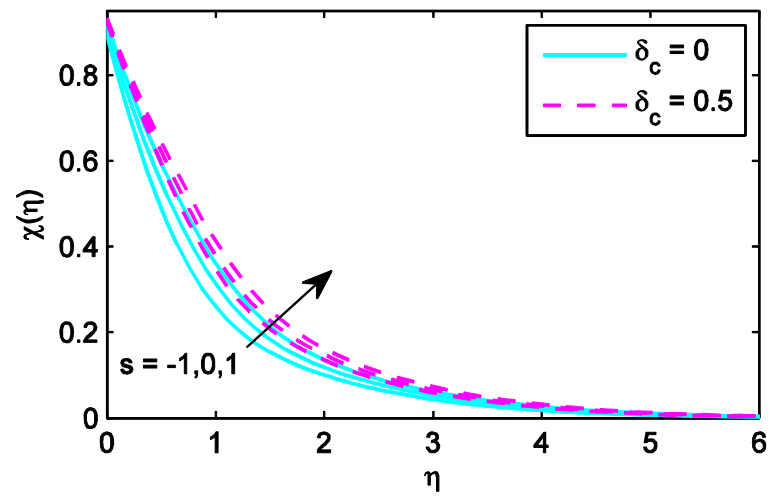

Figure 10: Effects of $s$ and $\delta_{\mathrm{c}}$ on $\phi(\eta)$ and $\chi(\eta)$

We now turn our attention to study the influence of microorganism slip parameter $\delta_{n}$ and Stefan blowing parameter, $s$ on the dimensionless radial velocity, temperature, microorganism and concentration. Figure 11a, b shows that greater blowing enhances the radial velocity and temperatures whereas suction induces the opposite effect. Higher microorganisms slip however has negligible effect on both velocity and temperature. It is clear from Fig. 12(b) that a higher microorganism slip parameter leads to a decrease of microorganism profiles. Note that the microorganism slip parameter $\delta_{n}$ also appears in the wall boundary condition (26) as $\chi(0)=1+\delta_{n} \chi^{\prime}(0)$. The coupling with the micro-organism density gradient i.e. $\chi \delta_{n} \chi^{\prime}(0)$ results in a delay in the diffusion of microorganisms from the disk surface to main body of nanofluid, similar to that induced by temperature and nanoparticle concentration slip effects. This delay results in opposition to microorganisms migrating from the disk (wall) to the free stream and manifests in a suppression in micro-organism species boundary layer thickness with $\delta_{n}$. The inclusion of micro-organism slip at the disk surface therefore produces results which are lower than those reported in no slip models.

(a)

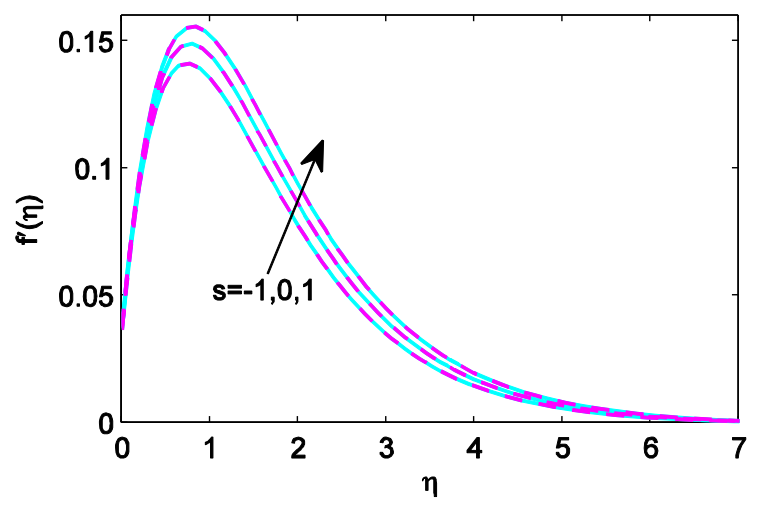

(b)

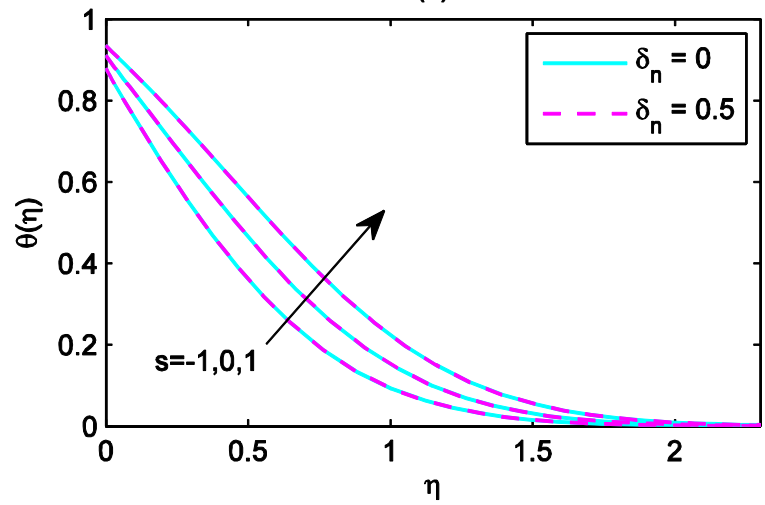

Figure 11: Effects of $s$ and $\delta_{n}$ on $\mathrm{f}^{\prime}(\eta)$ and $\theta(\eta)$ 
(a)

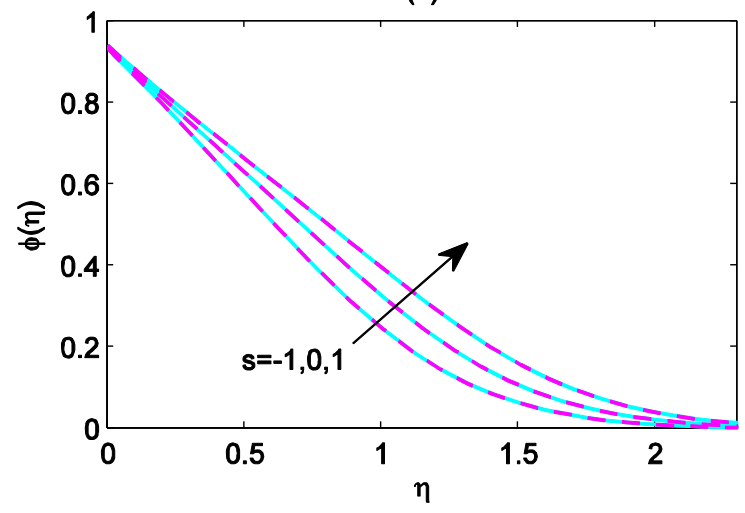

(b)

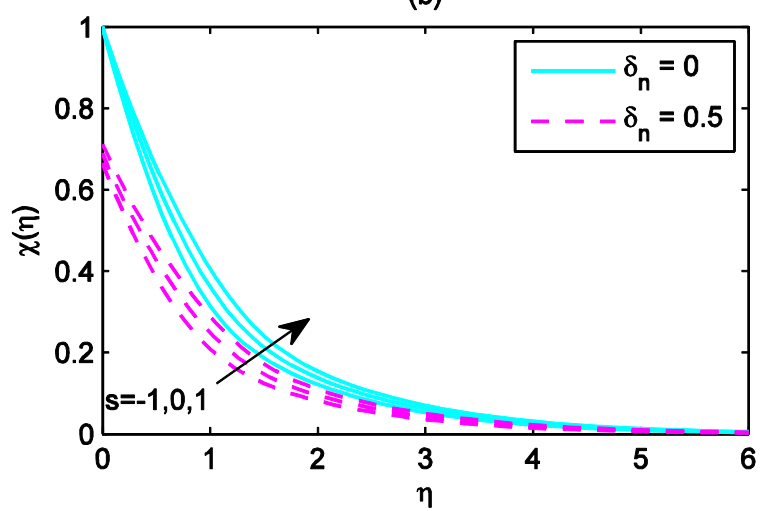

Figure 12: Effects of $s$ and $\delta_{\mathrm{n}}$ on $\phi(\eta)$ and $\chi(\eta)$

Inspection of Fig. 13 reveals that radial and circumferential (tangential) friction factors both decrease with elevation in the radial and circumferential momentum slip parameters in the presence or absence of blowing. Anisotropic slip therefore induces significant deceleration on the disk surface. Neglection of anisotropic slip in conventional mathematical models of swirling disk flow therefore produces overestimates of the surface skin friction components. It is also noteworthy that radial skin friction grows in a linear fashion with increasing Stefan blowing whereas generally there is a linear decay in circumferential skin friction factor with greater Stefan blowing.

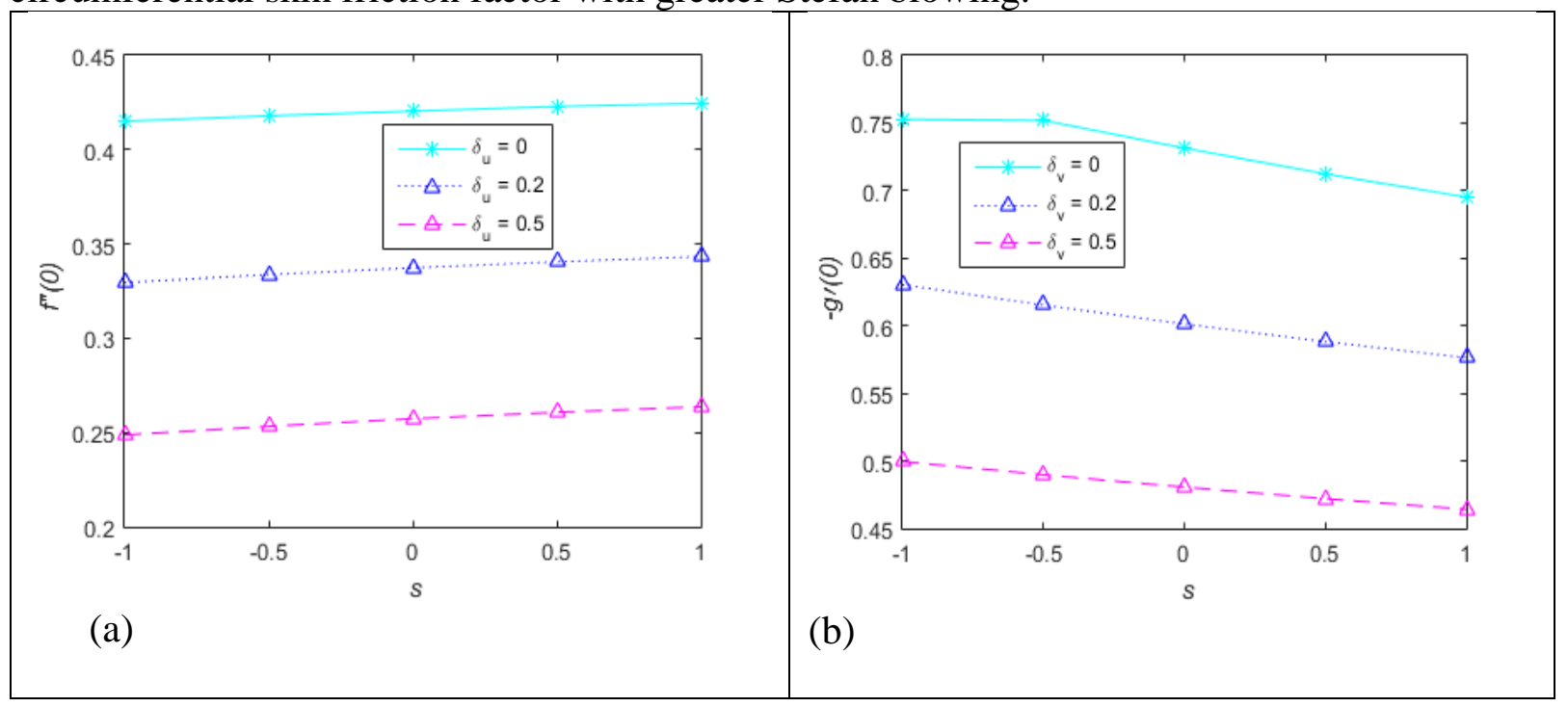

Figure 13: Influence of $s$ on (a) $f^{\prime \prime}(0)$ for different values of $\delta_{u}$, (b) - $g^{\prime}(0)$ for different values of $\delta_{\mathrm{v}}$.

Fig. 14 shows that disk surface heat transfer rate and nanoparticle mass transfer rate both decrease with higher thermal and nanoparticle mass slip parameters in the absence or presence of blowing. The inclusion of these slip effects therefore produces results which are significantly lower than no-slip model predictions and are thereby conservative for designers [73, 74]. With increased blowing, heat transfer rate and nanoparticle mass transfer rate are clearly depleted (linear decays) except for the case of strong nanoparticle mass slip (Fig. 14b, pink line) for which there is a weak linear growth in nanoparticle mass transfer rate. 


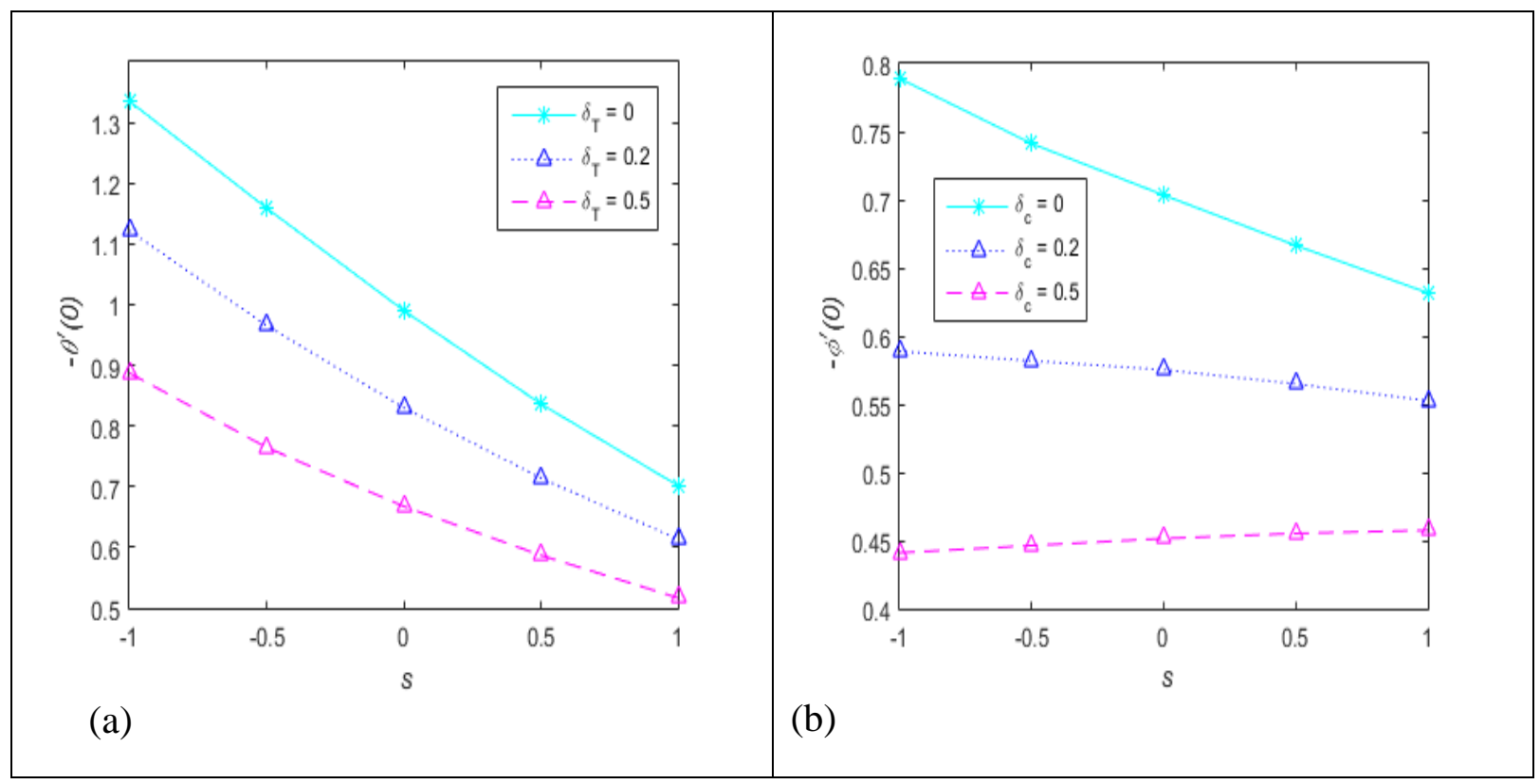

Figure 14: Impact of Stefan blowing, $s$, on (a) $-\theta^{\prime}(0)$ for different values of $\delta_{T}$, (b) on - $\phi^{\prime}(0)$ for different values of $\delta_{C}$.

Finally, Fig. 15 indicates that with an increase of microorganism slip parameters, for any Stefan blowing parameter, the microorganism transfer rate is substantially decreased. Maximum microorganism transfer rate at the disk surface therefore corresponds to the case of no microorganism $\operatorname{slip}\left(\delta_{n}=0\right)$.

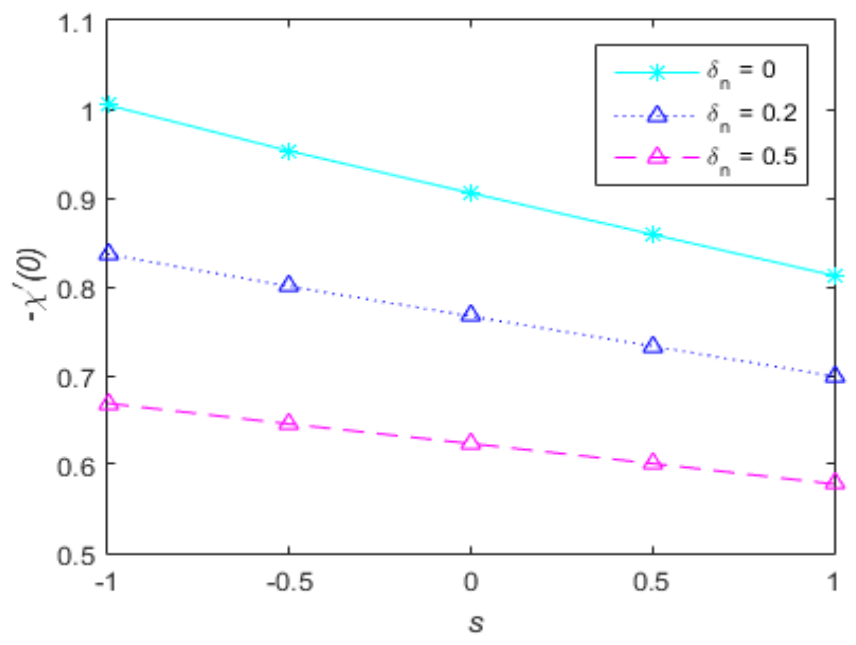

Figure 15: The plot of $-\chi^{\prime}(0)$ versus $s(-1<s<1)$ with different values of $\delta_{n}$

Solutions for radial and circumferential skin friction, heat transfer rate (Nusselt number function), nano-particle wall mass transfer rate (Sherwood number function) and wall motile micro-organism density number gradient are presented in Table 2 for various values of the Stefan blowing parameter $(s)$, disk surface slip parameters $\left(\delta_{u}, \delta_{v}, \delta_{T}, \delta_{c}, \delta_{n}\right)$, power-law stretching rate $(m)$ and Darcy number $(D a)$. Radial skin friction $\left(f^{\prime \prime}(0)\right)$ is significantly increased with a switch in power-law stretching from negative to positive values; however circumferential skin friction $\left(-g^{\prime}(0)\right)$ is decreased as are heat transfer rate $\left(-\theta^{\prime}(0)\right)$, nanoparticle mass transfer rate $\left(-\phi^{\prime}(0)\right)$ and micro-organism density number rate $(-$ $\chi^{\prime}(0)$ ). Disk radial stretching therefore exerts a significant influence on wall transport characteristics and may be exploited by designers to achieve desired results [74, 75]. The impact of the other parameters agrees with the profiles plotted in earlier figures. 
Table 2 (Part 1): Computational values of $f^{\prime \prime}(0),-g^{\prime}(0),-\theta^{\prime}(0),-\phi^{\prime}(0)$ and $-\chi^{\prime}(0)$ for different parameters: $s, \delta_{u}, \delta_{v}, \delta_{T}, \delta_{c}, \delta_{n}, m$ and $D a$.

\begin{tabular}{|c|c|c|c|c|c|c|c|c|c|c|c|c|}
\hline$s$ & $\delta_{u}$ & $\delta_{v}$ & $\delta_{T}$ & $\delta_{c}$ & $\delta_{n}$ & $m$ & $\mathrm{Da}$ & $f^{\prime}(0)$ & $-g^{\prime}(0)$ & $-\theta^{\prime}(0)$ & $-\phi^{\prime}(0)$ & $-\chi^{\prime}(0)$ \\
\hline-1 & \multirow{3}{*}{0} & 0.1 & 0.1 & 0.1 & 0.1 & -0.5 & 10 & 0.4151 & 0.6456 & 1.1192 & 0.6302 & 0.8583 \\
\hline 0 & & 0.1 & 0.1 & 0.1 & 0.1 & -0.5 & 10 & 0.4204 & 0.6118 & 0.8196 & 0.596 & 0.7833 \\
\hline 1 & & 0.1 & 0.1 & 0.1 & 0.1 & -0.5 & 10 & 0.4245 & 0.5835 & 0.5869 & 0.5558 & 0.7086 \\
\hline-1 & \multirow{3}{*}{0.5} & 0.1 & 0.1 & 0.1 & 0.1 & -0.5 & 10 & 0.2487 & 0.8038 & 1.4482 & 0.7726 & 1.0437 \\
\hline 0 & & 0.1 & 0.1 & 0.1 & 0.1 & -0.5 & 10 & 0.2573 & 0.7669 & 1.0931 & 0.721 & 0.9438 \\
\hline 1 & & 0.1 & 0.1 & 0.1 & 0.1 & -0.5 & 10 & 0.2637 & 0.7355 & 0.8102 & 0.6727 & 0.857 \\
\hline-1 & 0.1 & \multirow{3}{*}{0} & 0.1 & 0.1 & 0.1 & -0.5 & 10 & 0.4025 & 0.7529 & 1.2462 & 0.6797 & 0.8958 \\
\hline 0 & 0.1 & & 0.1 & 0.1 & 0.1 & -0.5 & 10 & 0.4141 & 0.7315 & 0.9347 & 0.6553 & 0.8575 \\
\hline 1 & 0.1 & & 0.1 & 0.1 & 0.1 & -0.5 & 10 & 0.4181 & 0.6952 & 0.6775 & 0.6104 & 0.7755 \\
\hline-1 & 0.1 & \multirow{3}{*}{0.5} & 0.1 & 0.1 & 0.1 & -0.5 & 10 & 0.269 & 0.4996 & 1.1014 & 0.5997 & 0.8227 \\
\hline 0 & 0.1 & & 0.1 & 0.1 & 0.1 & -0.5 & 10 & 0.2775 & 0.4806 & 0.8142 & 0.57 & 0.7524 \\
\hline 1 & 0.1 & & 0.1 & 0.1 & 0.1 & -0.5 & 10 & 0.2845 & 0.4642 & 0.589 & 0.5342 & 0.6827 \\
\hline-1 & 0.1 & 0.1 & \multirow{3}{*}{0} & 0.1 & 0.1 & -0.5 & 10 & 0.3689 & 0.6897 & 1.3351 & 0.5899 & 0.8423 \\
\hline 0 & 0.1 & 0.1 & & 0.1 & 0.1 & -0.5 & 10 & 0.375 & 0.6591 & 0.9903 & 0.6012 & 0.806 \\
\hline 1 & 0.1 & 0.1 & & 0.1 & 0.1 & -0.5 & 10 & 0.3803 & 0.6297 & 0.7017 & 0.584 & 0.7484 \\
\hline-1 & 0.1 & 0.1 & \multirow{3}{*}{0.5} & 0.1 & 0.1 & -0.5 & 10 & 0.3648 & 0.7092 & 0.8872 & 0.9525 & 1.1551 \\
\hline 0 & 0.1 & 0.1 & & 0.1 & 0.1 & -0.5 & 10 & 0.375 & 0.6591 & 0.6676 & 0.7191 & 0.8961 \\
\hline 1 & 0.1 & 0.1 & & 0.1 & 0.1 & -0.5 & 10 & 0.3805 & 0.6284 & 0.5168 & 0.6093 & 0.7612 \\
\hline-1 & 0.1 & 0.1 & 0.1 & \multirow{3}{*}{0} & 0.1 & -0.5 & 10 & 0.3667 & 0.7004 & 1.2763 & 0.789 & 1.0108 \\
\hline 0 & 0.1 & 0.1 & 0.1 & & 0.1 & -0.5 & 10 & 0.375 & 0.6591 & 0.9007 & 0.7034 & 0.8812 \\
\hline 1 & 0.1 & 0.1 & 0.1 & & 0.1 & -0.5 & 10 & 0.3806 & 0.6273 & 0.6365 & 0.6317 & 0.776 \\
\hline-1 & 0.1 & 0.1 & 0.1 & \multirow{3}{*}{0.5} & 0.1 & -0.5 & 10 & 0.3705 & 0.6819 & 1.118 & 0.441 & 0.7218 \\
\hline 0 & 0.1 & 0.1 & 0.1 & & 0.1 & -0.5 & 10 & 0.375 & 0.6591 & 0.9108 & 0.4515 & 0.6982 \\
\hline 1 & 0.1 & 0.1 & 0.1 & & 0.1 & -0.5 & 10 & 0.3792 & 0.6359 & 0.7136 & 0.4575 & 0.6708 \\
\hline
\end{tabular}

Table 2 (Part 2): Computational values of $f^{\prime \prime}(0),-g^{\prime}(0),-\theta^{\prime}(0),-\phi^{\prime}(0)$ and $-\chi^{\prime}(0)$ for different parameters: $s, \delta_{u}, \delta_{v}, \delta_{T}, \delta_{c}, \delta_{n}, m$ and $D a$.

\begin{tabular}{|c|c|c|c|c|c|c|c|c|c|c|c|c|}
\hline $\mathrm{s}$ & $\delta \mathrm{u}$ & $\delta \mathrm{v}$ & $\delta \mathrm{T}$ & $\delta \mathrm{c}$ & $\delta \mathrm{n}$ & $\mathrm{m}$ & $\mathrm{Da}$ & $f^{\prime \prime}(0)$ & $-g^{\prime}(0)$ & $-\theta^{\prime}(0)$ & $-\phi^{\prime}(0)$ & $-\chi^{\prime}(0)$ \\
\hline-1 & 0.1 & 0.1 & 0.1 & 0.1 & \multirow{3}{*}{0} & -0.5 & 10 & 0.368 & 0.694 & 1.2218 & 0.6707 & 1.0046 \\
\hline 0 & 0.1 & 0.1 & 0.1 & 0.1 & & -0.5 & 10 & 0.375 & 0.6591 & 0.9034 & 0.633 & 0.9055 \\
\hline 1 & 0.1 & 0.1 & 0.1 & 0.1 & & -0.5 & 10 & 0.3803 & 0.6294 & 0.6545 & 0.5905 & 0.8126 \\
\hline-1 & 0.1 & 0.1 & 0.1 & 0.1 & 0.5 & -0.5 & 10 & 0.368 & 0.694 & 1.2218 & 0.6707 & 0.6687 \\
\hline
\end{tabular}




\begin{tabular}{|c|c|c|c|c|c|c|c|c|c|c|c|c|}
\hline 0 & 0.1 & 0.1 & 0.1 & 0.1 & & -0.5 & 10 & 0.375 & 0.6591 & 0.9034 & 0.633 & 0.6233 \\
\hline 1 & 0.1 & 0.1 & 0.1 & 0.1 & & -0.5 & 10 & 0.3803 & 0.6294 & 0.6545 & 0.5905 & 0.5778 \\
\hline-1 & 0.1 & 0.1 & 0.1 & 0.1 & 0.1 & \multirow{3}{*}{-0.5} & 10 & 0.3679 & 0.694 & 1.2215 & 0.6705 & 0.9129 \\
\hline 0 & 0.1 & 0.1 & 0.1 & 0.1 & 0.1 & & 10 & 0.3749 & 0.659 & 0.9032 & 0.6328 & 0.8304 \\
\hline 1 & 0.1 & 0.1 & 0.1 & 0.1 & 0.1 & & 10 & 0.3802 & 0.6293 & 0.6544 & 0.5903 & 0.7518 \\
\hline-1 & 0.1 & 0.1 & 0.1 & 0.1 & 0.1 & \multirow{3}{*}{0.5} & 10 & 0.4831 & 0.577 & 1.1545 & 0.6373 & 0.8723 \\
\hline 0 & 0.1 & 0.1 & 0.1 & 0.1 & 0.1 & & 10 & 0.4956 & 0.5423 & 0.8534 & 0.6074 & 0.7999 \\
\hline 1 & 0.1 & 0.1 & 0.1 & 0.1 & 0.1 & & 10 & 0.5052 & 0.5129 & 0.6156 & 0.5701 & 0.7277 \\
\hline-1 & 0.1 & 0.1 & 0.1 & 0.1 & 0.1 & -0.5 & \multirow{3}{*}{2} & 0.3189 & 0.7746 & 1.1299 & 0.6063 & 0.8293 \\
\hline 0 & 0.1 & 0.1 & 0.1 & 0.1 & 0.1 & -0.5 & & 0.3252 & 0.742 & 0.8368 & 0.5704 & 0.7516 \\
\hline 1 & 0.1 & 0.1 & 0.1 & 0.1 & 0.1 & -0.5 & & 0.3301 & 0.7144 & 0.6072 & 0.532 & 0.679 \\
\hline-1 & 0.1 & 0.1 & 0.1 & 0.1 & 0.1 & -0.5 & \multirow{3}{*}{10} & 0.368 & 0.694 & 1.2218 & 0.6707 & 0.9129 \\
\hline 0 & 0.1 & 0.1 & 0.1 & 0.1 & 0.1 & -0.5 & & 0.375 & 0.6591 & 0.9034 & 0.633 & 0.8303 \\
\hline 1 & 0.1 & 0.1 & 0.1 & 0.1 & 0.1 & -0.5 & & 0.3802 & 0.6293 & 0.6544 & 0.5903 & 0.7518 \\
\hline
\end{tabular}

\section{CONCLUSIONS}

Motivated by new developments in rotating disk membrane oxygenators and rotating bioreactor designs, a mathematical model has been presented for viscous, incompressible, steady-state swirling transport from an impermeable rotating disk immersed in porous medium saturated with water-based nanofluid containing gyrotactic microorganisms. The disk rotates at a constant angular velocity and is able to radially stretch, according to a power-law relationship, and Darcy's model is employed for simulating porous medium drag effects. Furthermore, anisotropic slip boundary conditions and blowing effects at the disk surface was considered. The governing conservation equations were converted to a set of similarity differential equations (SODEs) using appropriate transformations. The SODEs were solved by the Chebyshev collocation method. Validation with earlier studies was made. The principal findings may be stated as follows:

- Radial velocity increases whereas temperature, concentration and microorganism density number decrease with Darcy number and radial slip parameter.

- Temperature is reduced with increasing thermal slip and nanoparticle concentration is suppressed with increasing wall mass slip.

- Micro-organism density number increases with the greater microorganism slip.

- Radial velocity, nanoparticle concentration, temperature and microorganism density are all enhanced with an increase in the value of the Stefan blowing parameter.

- Radial skin friction is strongly elevated with a positive power-law stretching exponent whereas it is reduced with negative values; the opposite behaviour is computed for circumferential skin friction, heat transfer rate, nanoparticle mass transfer rate and micro-organism density number rate.

- Radial and circumferential skin friction, heat transfer rate, nanoparticle mass transfer rate and micro-organism density number rate are all reduced generally with higher slip parameters, irrespective of the presence of Stefan blowing.

The present work was confined to steady-state flow. Future studies will consider time-dependent flows which are also of relevance to rotating hybrid membrane oxygenator systems and will be communicated imminently. 


\section{ACKNOWLEDGEMENTS}

This work was supported by Fundamental Research Grant Scheme (FRGS) No. FRGS/1/2019/ICT02/UMP/02/5 from Ministry of Higher Education, Malaysia. Professor O. Anwar Bég wishes to express his deep gratitude to Miss Sootina Sioux Bég (2008-2020) for her wonderful companionship; she will be greatly missed. The authors are also grateful to the reviewers for some of their comments which have improved the present work.

\section{REFERENCES}

[1] Minkowycz, W.J., Sparrow, E.M. and Abraham, J.P. (Eds), (2013), Nanoparticle Heat Transfer and Fluid Flow, CRC Press, Taylor and Francis Group, New York, USA.

[2] E. Zarifi, G. Jahanfarnia, F. Veysi, Subchannel analysis of nanofluids application to VVER-1000 reactor, Chemical Engineering Research and Design, 91 (2013) 625-632.

[3] O. Gravel, J. Lauzon-Gauthier, Carl Duchesne, Faïçal Larachi, Inception of vortical coherent structures from spinning magnetic nanoparticles in rotating magnetic fields - New nanofluid microscale mixing tool, Chemical Engineering J., 260 (2015) 338-346.

[4] D. A. Nield and A. Bejan, Convection in Porous Media, Fifth Edition, Springer (2017).

[5] Choi SUS. Enhancing thermal conductivity of fluids with nanoparticles, The Proceedings of the 1995. ASME International Mechanical Engineering Congress and Exposition, San Francisco, USA, ASME 1995: 99-105.

[6] J. Buongiorno, Convective transport in nanofluids, ASME Journal of Heat Transfer, (2006), 128(3):240-250.

[7] S. K. Das, S. U. S. Choi, W. Yu, T. Pradeep, Nanofluids: Science and Technology, John Wiley, New York (2007).

[8] M.M. Rahman, A. V Rosca, I. Pop, Boundary layer flow of a nanofluid past a permeable exponentially shrinking/stretching surface with second order slip using Buongiorno's model, Int. J. Heat Mass Transf. 77 (2014) 1133-1143.

[9] N. Acharya, K. Das, P. K. Kundu, Outlining the impact of second-order slip and multiple convective condition on nanofluid flow: A new statistical layout, Can. J. Phys. 96(2018) 104-111.

[10] B. Mahanthesh, B.J. Gireesha, R. Subba, R. Gorla, Unsteady three-dimensional MHD flow of a nano Eyring-Powell fluid past a convectively heated stretching sheet in the presence of thermal radiation, viscous dissipation and Joule heating, J. Assoc. Arab Univ. Basic Appl. Sci. 23 (2017) 7584. doi:10.1016/j.jaubas.2016.05.004.

[11] Hwang, Y., and Pedley, T. J., Stability of downlowing gyrotactic microorganism suspensions in a two-dimensional vertical channel, Journal of Fluid Mechanics, 749, 750-777, 2014

[12] H. Tsai, D.-S. Liou, L.-S. Kuo, P.-H. Chen, Rapid mixing between ferro-nanofluid and water in a semi-active Y-type micromixer, Sensors Actuators A. 153 (2009) 267-273. doi:10.1016/j.sna.2009.05.004.

[13] H. Li, S. Liu, Z. Dai, J. Bao, X. Yang, Applications of nanomaterials in electrochemical enzyme biosensors, Sensors. 9 (2009) 8547-8561. doi:10.3390/s91108547. 
[14] Munir, J. Wang, H.S. Zhou, Dynamics of capturing process of multiple magnetic nanoparticles in a flow through microfluidic bioseparation system, IEEE Nanobiotechnology. 3 (2009) 55. doi:10.1049/iet-nbt.2008.0015.

[15] Y. Chisti, Biodiesel from microalgae, Biotechnol. Adv. 25 (2007) 294-306.

[16] K. G. Satyanarayana, A. B. Mariano and J. V. C. Vargas, A review on microalgae, a versatile source for sustainable energy and materials, Int. J. Energy Research 35 (2011) 291-311

[17] M. Aneja, O. Anwar Bég, Sapna Shamra and S. Kuharat, Computation of electroconductive gyrotactic bioconvection under nonuniform magnetic field: Simulation of smart bio-nanopolymer coatings for solar energy, int. J. Modern Physics B (2020). DOI: 10.1142/S0217979220500289

[18] O. P. Roncallo et al., Comparison of two different vertical column photobioreactors for the cultivation of nannochloropsis sp., ASME J. Energy Resour. Technol. 135 (2012) 011201-011201-7.

[19] H. Xu, Lie group analysis of a nanofluid bioconvection flow past a vertical flat surface with an outer power-law stream, ASME J. Heat Transfer. 137 (2015) 041101 (1-9).

[20] Raees, H. Xu, S.-J. Liao, Unsteady mixed nano-bioconvection flow in a horizontal channel with its upper plate expanding or contracting, Int. J. Heat Mass Transf. 86 (2015) 174-182.

[21] S. Siddiqa, G.E. Hina, N. Begum, S. Saleem, M.A. Hossain, R. Subba, R. Gorla, Numerical solutions of nanofluid bioconvection due to gyrotactic microorganisms along a vertical wavy cone, Int. J. Heat Mass Transf. 101 (2016) 608-613.

[22] M.E. Taskin et al., Micro-scale modeling of flow and oxygen transfer in hollow fiber membrane bundle, Journal of Membrane Science vol. 362,1-2 (2010): 172-183.

[23] F. Gerbode, J. J. Osborn, J. Bruce Johnston, Experience with perfusion hypothermia using an improved rotating disc oxygenator, Thorax (1960), 15, 185

[24] M. Gaber, M.A.A. Mohamed, The effects of radiation and Hall current on the unsteady mhd laminar convective fluid flow through porous medium due to a porous rotating disk, Int. J. Theor. Appl. Multiscale Mech. 4 (2009).

[25] T. V. Kármán, Uber laminare und turbulent Reibung (on laminar and turbulent flow). ZAMM, (1921), 1:233-252.

[26] V. Shevchuk, Convective Heat and Mass Transfer in Rotating Disk Systems, Springer,New York (2009).

[27] S. R. Mishra, M. Shamshuddin, O. Anwar Bég, Ali Kadir, Numerical study of heat transfer and viscous flow in a dual rotating extendable disk system with a non-Fourier heat flux model, Heat Transfer - Asian Research, 48 (1), 435-459 (2019).

[28] O. Anwar Bég, W. S. Jouri, Tasveer A. Bég, Ali Kadir, H. J. Leonard, M.D. Shamshuddin and T.K. Hung, Computation of asymmetric micropolar flow in a bionic prosthetic dual disk system with blowing (injection) and Reynolds number effects, Int. Conf. Advances in Mathematics, Physics \& Applied Science (ICAMPA), New Delhi, India, March $1^{\text {st }}(2020)$.

[29] C. Yin, L. Zheng, C. Zhang, X. Zhang, Flow and heat transfer of nanofluids over a rotating disk with uniform stretching rate in the radial direction, Propuls. Power Res. 6 (2017) 25-30.

[30] O. Anwar Bég, F. Mabood and M. Nazrul Islam, Homotopy simulation of nonlinear unsteady 
rotating nanofluid flow from a spinning body, International Journal of Engineering Mathematics, Volume 2015, Article ID 272079, 15 pages (2015).

[31] Ali Kadir, S.R. Mishra, M. Shamshuddin and O. Anwar Bég, Numerical simulation of Von Karman swirling bioconvection nanofluid flow from a deformable rotating disk, ICHTFM 2018: 20th International Conference on Heat Transfer and Fluid Mechanics, WASET, Istanbul, Turkey, August $16-17$ (2018).

[32] Vafai, K. Porous Media: Applications in Biological Systems and Biotechnology, CRC Press, Florida, USA (2010).

[33] Khaled, A. R. A. and Vafai, K. The role of porous media in modeling flow and heat transfer in biological tissues. International Journal of Heat and Mass Transfer, 46(26), 4989-5003 (2003)

[34] B. Podola et al., Porous substrate bioreactors: a paradigm shift in microalgal biotechnology? Trends in Biotechnology, 35, 121-132 (2017).

[35] A. Yücel, Heat and mass transfer about vertical surfaces in saturated porous media, AIChE Symp. Ser. 269 (85) (1989) 344.

[36] F.C. Lai, F.A. Kulacki, Coupled heat and mass transfer by natural convection from vertical surfaces in porous media, Int. J. Heat Mass Transf. 34 (4-5) (1991) 1189-1194.

[37] O. Anwar Bég, Sandile S. Motsa, A. Kadir, Tasveer A. Bég and M.N. Islam, Spectral quasilinear numerical simulation of micropolar convective wall plumes in high permeability porous media, $J$. Engineering Thermophysics, 25 (4) 1-24 (2016).

[38] J.C. Umavathi and O. Anwar Bég, Numerical study of double-diffusive dissipative reactive convective flow in an open vertical duct containing a non-Darcy porous medium with Robin boundary conditions, J. Engineering Mathematics (2019). https://doi.org/10.1007/ s10665-019-10022-w (13 pages)

[39] O. Anwar Bég, Tasveer A. Bég, R. Bhargava, S. Rawat and D. Tripathi, Finite element study of pulsatile magneto-hemodynamic non-Newtonian flow and drug diffusion in a porous medium channel, J. Mechanics in Medicine and Biology, 12 (4) 1250081.1 - 1250081.26 (2012).

[40] H.A. Attia, Steady flow over a rotating disk in porous medium with heat transfer, Non-linear Anal. Model. Control. 14 (2009) 21-26.

[41] O. Anwar Bég, K. Abdel Malleque and M.N. Islam, Modelling of Ostwald-deWaele nonNewtonian flow over a rotating disk in a non-Darcian porous medium, Int. J. Applied Mathematics and Mechanics, 8 (13): 46-67, 2012. (2012).

[42] T. Hayat, F. Haider, T. Muhammad and A. Alsaedi, On Darcy-Forchheimer flow of carbon nanotubes due to a rotating disk, International Journal of Heat and Mass Transfer, 112, 248-254 (2017).

[43] B. Porter, R. Zauel, H. Stockman, R. Guldberg and D. Fyhrie, 3-D computational modeling of media flow through scaffolds in a perfusion bioreactor, Journal of Biomechanics, 38, 543-549 (2005).

[44] H. Singh, et al., Flow modelling in a novel non-perfusion conical bioreactor, Biotechnol. Bioeng, 97, 1291-1299, (2007).

[45] D. Tripathi, O. Anwar Bég and J. Curiel-Sosa, Homotopy semi-numerical simulation of peristaltic 
flow of generalized Oldroyd-B fluids with slip effects, Computer Methods in Biomechanics Biomedical Engineering, 17(4) (2014) 433-442.

[46] A.A. Avramenko, N.P. Dmitrenko, I.V. Shevchuk, A.I. Tyrinov, V.I. Shevchuk, Heat transfer of incompressible flow in a rotating microchannel with slip boundary conditions of second order, International Journal of Numerical Methods for Heat \& Fluid Flow, 29 (5), 1786-1814 (2019).

[47] Lienhard, J. H. IV, and Lienhard, J. H. V, A Heat Transfer Textbook, $3^{\text {rd }}$ ed., Phlogiston Press, Cambridge, MA, pp. 662-663 (2005).

[48] K. Schwartz et al., The use of drip flow and rotating disk reactors for staphylococcus aureus biofilm analysis, J. Vis. Exp., (46): 2470 (2010).

[49] M.R. Hajmohammadi, H. Maleki, G. Lorenzini, S.S. Nourazar, Effects of $\mathrm{Cu}$ and Ag nanoparticles on flow and heat transfer from permeable surfaces, Adv. Powder Technol. 26 (2015) 193199.

[50] N.A. Latiff, M.J. Uddin, A.I.M. Ismail, Stefan blowing effect on bioconvective flow of nanofluid over a solid rotating stretchable disk, Propuls. Power Res. 5 (2016) 267-278.

[51] Zohra, F. T., M. J. Uddin, A. I. M. Ismail, O. Anwar Beg, and Ali Kadir. Anisotropic slip magnetobioconvection flow from a rotating cone to a nanofluid with Stefan blowing effects, Chinese Journal Physics 56, no. 1 (2018): 432-448.

[52] M.J. Uddin, M.N. Kabir, O.Anwar Bég, Computational investigation of Stefan blowing and multiple-slip effects on buoyancy-driven bioconvection nanofluid flow with microorganisms, Int. $J$. Heat Mass Transfer. 95 (2016) 116-130.

[53] Ragui, K., Boutra, A., Bennacer, R., Labsi, N., \& Benkahla, Y. K. Correlating heat and mass transfer coefficients for thermosolutal convection within a porous annulus of a circular shape: case of internal pollutants spreading. Heat and Mass Transfer, 54 (7), 2061-2078 (2018).

[54] O. Anwar Bég, Numerical methods for multi-physical magnetohydrodynamics, Chapter 1, pp. 1112, New Developments in Hydrodynamics Research, M. J. Ibragimov and M. A. Anisimov, Eds., Nova Science, New York, USA (2012).

[55] C. Canuto, M.Y. Hussaini, A. Quarteroni, T.A. Zang, Spectral Methods in Fluid Dynamics, Springer-Verlag, New York, USA (1988).

[56] Kaladhar, K., Reddy, K. M., \& Srinivasacharya, D., Inclined magnetic field, thermal radiation and Hall current effects on mixed convection flow between vertical parallel plates. ASME Journal of Heat Transfer, 141(10): 102501 (2019).

[57] F.B. Belgacem, M. Grundmann, Approximation of the wave and electromagnetic diffusion equations by spectral methods, SIAM Journal on Scientific Computing 20 (1) (1998), 13-32.

[58] Ma'en, S. S., \& Butcher, E. A. (2012). Free vibration analysis of non-rotating and rotating Timoshenko beams with damaged boundaries using the Chebyshev collocation method. International Journal of Mechanical Sciences, 60(1), 1-11.

[59] Aly, E. H., \& Sayed, H. M. (2017). Magnetohydrodynamic and thermal radiation effects on the boundary-layer flow due to a moving extensible surface with the velocity slip model: A comparative study of four nanofluids. Journal of Magnetism and Magnetic Materials, 422, 440-451. 
[60] Agarwal, P., and A. A. El-Sayed. Non-standard finite difference and Chebyshev collocation methods for solving fractional diffusion equation. Physica A: Statistical Mechanics and Its Applications, 500 (2018): 40-49.

[61] Uddin, M. J., Kabir, M. N., Bég, O. A., \& Alginahi, Y. (2018). Chebyshev collocation computation of magneto-bioconvection nanofluid flow over a wedge with multiple slips and magnetic induction. Proceedings of the Institution of Mechanical Engineers, Part N: Journal of Nanomaterials, Nanoengineering and Nanosystems, 232(4), 109-122.

[62] Tian, X. Y., Li, B. W., \& Hu, Z. M. (2018). Convective stagnation point flow of a MHD nonNewtonian nanofluid towards a stretching plate. International Journal of Heat and Mass Transfer, 127, 768-780.

[63] Sun, Y. S., Ma, J., \& Li, B. W. (2012). Chebyshev collocation spectral method for threedimensional transient coupled radiative-conductive heat transfer. ASME Journal of Heat Transfer, 134(9), 092701.

[64] Vasu, B., RamReddy, C., Murthy, P. V. S. N., \& Gorla, R. S. R. (2017). Entropy generation analysis in non-linear convection flow of thermally stratified fluid in saturated porous medium with convective boundary condition. ASME Journal of Heat Transfer, 139(9), 091701.

[65] Bai, Z. Q., Gu, Y., \& Fan, C. M. (2019). A direct Chebyshev collocation method for the numerical solutions of three-dimensional Helmholtz-type equations. Engineering Analysis with Boundary Elements, 104, 26-33.

[66] O. Anwar Bég, M. Hameed and T.A. Bég, Chebyschev spectral collocation simulation of nonlinear boundary value problems in electrohydrodynamics, Int. J. Computational Methods in Engineering Science and Mechanics, 14, 104-115 (2013).

[67] MD. Shamshuddin, M. Ferdows, Rezwan, O. Anwar Bég, A. Kadir, Ferromagnetic and nonmagnetic nanoparticles in nanofluid flow from a stretching cylinder with magnetic induction: spectral relaxation solution, Proceedings of the International Conference on Numerical Heat Transfer and Fluid Flow (NHTFF-2020), NIT Warangal, India - Jan 17-19 (2020).

[68] O. Anwar Bég, Nonlinear multi-physical laminar nanofluid bioconvection flows: Models and computation, A. Sohail, Z. Li (Eds.): Computational Approaches in Biomedical Nano-Engineering, Wiley, Chapter 5, pp. 113-145 (2018).

[69] Chen, J. Chen, Y. Geng, K. Chen, Three-dimensional boundary layer flow over a rotating disk with power-law stretching in a nanofluid containing gyrotactic microorganisms, Heat Transf. - Asian Res. (2017) 1-14. doi:10.1002/htj.21327

[70] T. Fang, Flow and mass transfer for an unsteady stagnation-point flow over a moving wall considering blowing effects, ASME J. Fluids Eng 136(7), 071103 (May 06, 2014) (7 pages) Paper No: FE-13-1342; doi: 10.1115/1.4026665.

[71] G. Hansen, Similarity Analysis of Boundary Value Problems in Engineering, Prentice-Hall, New Jersey, USA (1964).

[72] O. Chtioui et al., Rotating discs bioreactor, a new tool for lipopeptides production, Process Biochemistry, 47, 2020-2024 (2012).

[72] R.W. Melchior, Evolution of membrane oxygenator technology for utilization during pediatric cardiopulmonary bypass, Pediatric Health Med Ther. 7: 45-56 (2016). 
[74] Qiu F, Guan Y, Su X, et al. Evaluation of neonatal membrane oxygenators with respect to gaseous microemboli capture and transmembrane pressure gradients. Artif Organs. 34:923-929 (2010).

[75] Drummond M, Braile DM, Lima-Oliveira AM, Camim AS, Oyama RS, Sandoval GH. Technological evolution of membrane oxygenators. Braz J Cardiovasc Surg. 20(4):432-437 (2005). 